\title{
DRBEM formulation for transient Stokes flow with slip boundary condition
}

\author{
S. Gümgüm ${ }^{\mathrm{a}, 1}$, Luiz C. Wrobel ${ }^{*, \mathrm{~b}, 2}$ \\ ${ }^{a}$ Department of Mathematics, Izmir University of Economics, Izmir, 35330, Turkey \\ ${ }^{b}$ Institute of Materials and Manufacturing, Brunel University London, Uxbridge, UB8 3PH, UK
}

\begin{abstract}
In this study, the effect of linear and nonlinear slip boundary conditions on the flow of a slow viscous fluid is investigated numerically. The boundary integral representation of the transient Stokes equations is given in primitive variables form. The fundamental solution to the steady Stokes equations is employed in the boundary element method (BEM) formulation. The time derivative is taken to the boundary with the dual reciprocity method and approximated by the finite difference method (FDM) until a steady-state is achieved. It is assumed that the fluid is capable of slip, with the slip velocity expressed as a function of shear rate at the wall. In the numerical tests, the fluid is initially assumed to be stationary; at each time step, the velocity boundary conditions along the walls are updated as the shear forces vary with time.
\end{abstract}

Key words: DRBEM, Stokes flow, Slip condition, BEM

\section{Introduction}

The area of micro and nanofluidics is fundamentally important due to the need of understanding the nature of fluid flow at these scales [1]. It has been demonstrated that, at these scales, the mechanical properties at the fluid-solid interface cannot be understood by extrapolating known properties of the bulk fluid [2]. An example of the breakdown of conventional macroscopic ideas at small scales is the no-slip boundary condition between a fluid and a solid, which is a fundamental notion in fluid mechanics [3]. Experimental [4, 5], theoretical [6] and numerical $[7,8]$ simulations at micro/nano scales have provided clear evidence that wall slip occurs at fluid-solid interfaces, and show that the degree of boundary slip is a function of the liquid viscosity and the shear rate. Variation in slip length arises from the fact that, during a collision with a solid surface, a fluid molecule will transfer some of its tangential momentum to the solid. The collision frequency is not high enough to ensure thermodynamic equilibrium, and a certain degree of slip tangential velocity must be allowed $[9]$.

\footnotetext{
*Corresponding author

Email addresses: sevin.gumgum@ieu.edu.tr (S. Gümgüm), luiz.wrobel@brunel.ac.uk (Luiz C. Wrobel)

${ }^{1}$ Tel.: +90-232-4888307

${ }^{2}$ Tel.: $+44(0) 1895266696$
} 
Luo and Pozrikidis [10] developed a BEM formulation for studying slip flow over a spherical particle in an infinite fluid and near a plane wall. In the case of a wall-bounded flow, the numerical model was axisymmetric and thus reduced to a one-dimensional integral equation in cylindrical coordinates. Ding and Ye [11] solved oscillatory slip Stokes flow problems by using a system of integral equations for the surface velocity and the normal derivative of its tangential component. The resulting integral equation for the normal derivative contains singularities of the Cauchy and Hadamard (hypersingular) types. Frangi et al. [12] employed a combined velocity-surface traction integral equation to study fluid damping in micro-electro-mechanical systems (MEMS), which also contains Cauchy and Hadamard singularities. Nieto et al. [15] developed a BEM formulation to study linear slip flow in rotating mixers, based on the use of the normal and tangential projections of the velocity integral equation, resulting in a weakly-singular mixed system of integral equations for the normal and tangential components of the surface traction. Later, their formulation was extended to incorporate the nonlinear slip condition [16]. Myong et al. [17] investigated slip flows in concentric rotating cylinders using a slip model defined in terms of the Langmuir adsorption isotherm for the gas-solid surface molecular interaction, instead of the Navier accommodation coefficient. They showed that despite the conceptual difference in the two slip models, both are in qualitative agreement with Monte Carlo simulation data in capturing the general features of the flow field.

In comparison with linear slip flows, research on nonlinear slip flows is more limited, with relatively few works reported in the literature. Newtonian flows with linear and nonlinear slip boundary condition were studied analytically by Matthews and Hill [6] for simple pressure driven flows. They observed that although the generalized Navier boundary condition is highly nonlinear in terms of the assumed form of solution, the integration constants obtained are still unique. Numerical solutions of nonlinear slip flows using Thompson and Troian's model are reported, where lid-driven polar cavity flow and thin film flow of a fluid between two spheres are considered in [18] and [19]. In a recent study, Power et al. [9] studied the effect of the Thompson and Troian's nonlinear slip condition on Couette flows between concentric rotating cylinders. They showed that, by using this type of nonlinear slip condition, it is possible to predict complex characteristics of the flow field not previously reported in the literature.

In this study, transient Stokes flow in different geometries with linear and nonlinear slip boundary conditions is numerically investigated using the Dual Reciprocity Boundary Element Method (DRBEM). The DRBEM for time-dependent Stokes flows was originally developed by Power and Partridge [20], considering standard no-slip boundary conditions. The technique gives rise to a system of first-order ordinary differential equations which is solved here by the Finite Difference Method (FDM). The method is initially applied to the pressure-driven flow in a channel, for which analytical solutions are available, and then extended to study flow past a step and flow in a square cavity.

\section{Governing Equations}

Fluid flow at micro and nano scales is characterized by very low values of the Reynolds number. In these cases, the non-linear convective terms in the Navier-Stokes equations can be neglected. The transient Stokes system of equations can be written in dimensionless form 
as $[21]$

$$
\begin{aligned}
& \frac{\partial u_{i}}{\partial t}=-\frac{\partial p}{\partial x_{i}}+\frac{\partial^{2} u_{i}}{\partial x_{j} \partial x_{j}} \\
& \frac{\partial u_{i}}{\partial x_{i}}=0
\end{aligned}
$$

where $u_{i}$ and $p$ are the components of the fluid velocity and pressure, respectively. The above equations are normalized by a characteristic length scale $L$ and a characteristic velocity scale $U$, respectively. At micro-scale conditions, $L$ is of the order of $100 \mu m$ while $U$ is of the order of $1 \mathrm{~mm} / \mathrm{s}$. Eq. (1) is supplemented by the initial conditions $u\left(x_{1}, x_{2}, 0\right)=u_{0}\left(x_{1}, x_{2}\right)$ and by velocity and surface force boundary conditions.

The Navier slip boundary condition states that the relative tangential fluid velocity, $u_{t}^{f}$, with respect to the tangential wall velocity, $U_{t}^{w}$, is proportional to the tangential projection of the local shear rate [3], [13, 14, 15],

$$
u_{t}^{f}-U_{t}^{w}=L s \dot{\gamma}_{t}
$$

where $L s$ is the slip length, which represents the hypothetical distance at the wall needed to satisfy the no-slip condition [1]. The variable $\dot{\gamma}_{t}$ is the local shear rate projection in the tangential direction defined as

$$
\dot{\gamma}_{t}=\left(\frac{\partial u_{i}}{\partial x_{j}}+\frac{\partial u_{j}}{\partial x_{i}}\right) n_{j} s_{i}=\left(\frac{\partial u_{t}}{\partial n}+\frac{\partial u_{n}}{\partial s}\right)
$$

where $n_{i}$ and $s_{i}$ are the $i$-th component of the normal and tangential vectors to a boundary surface showing the outward and the counter-clockwise direction, respectively.

The slip length, $L s$, can be either linear or nonlinear. The nonlinear slip length also depends on the tangential shear rate at the solid surface, given as [16]

$$
L s=\frac{b_{0}}{\sqrt{\left(1-\beta \dot{\gamma}_{t}\right)^{n}}}
$$

where $\beta=1 / \dot{\gamma}_{c}, \dot{\gamma}_{c}$ is the critical shear rate, $b_{0}$ is the slip length in the case of linear slip condition, and $n$ is an index which depends on the cohesive property at the interface. The nonlinear slip boundary condition is obtained by substituting Eq. (4) in Eq. (2). The constant slip length and the no-slip condition are the limiting cases of Eq. (4) [16]. The first one refers to the condition when $\dot{\gamma}_{c} \rightarrow \infty(\beta \rightarrow 0)$ for finite values of $b_{0}$, corresponding to the limit $L s \rightarrow b_{0}$ (constant slip length). On the other hand, the limiting case when $n \rightarrow \infty$, for finite values of $\dot{\gamma}_{c}$ and $b_{0}$, corresponds to $L s \rightarrow 0$, resulting in the classical no-slip condition.

\section{Boundary Element Formulation}


The Stokes velocity field has the following direct integral representation formulae for an arbitrary point $\mathbf{x}$ in a closed domain $\Omega$ bounded by a closed surface $\Gamma[21]$ :

$$
c(\mathbf{x}) u_{k}(\mathbf{x})-\int_{\Gamma} K_{k j}(\mathbf{x}, \mathbf{y}) u_{j}(\mathbf{y}) d \Gamma_{\mathbf{y}}+\int_{\Gamma} u_{j}^{k}(\mathbf{x}, \mathbf{y}) t_{j}(\mathbf{y}) d \Gamma_{\mathbf{y}}=\int_{\Omega} u_{j}^{k}(\mathbf{x}, \mathbf{y}) g_{j}(\mathbf{y}) d \Omega_{\mathbf{y}}
$$

where $t_{j}$ are the components of the surface traction, $g_{j}=\frac{\partial u_{j}}{\partial t}$, and $c(\mathbf{x})=\frac{1}{2}$ for a point located on a smooth part of the boundary and 1 when the point is on the domain. For any boundary point $c(\mathbf{x})=\frac{\theta}{2 \pi}$ where $\theta$ is the internal angle at the considered point in radians.

The fundamental velocity and traction for two-dimensional problems are given by [21]

$$
\begin{aligned}
& u_{i}^{j}(\mathbf{x}, \mathbf{y})=-\frac{1}{4 \pi}\left[\ln \left(\frac{1}{r}\right) \delta_{i j}+\frac{\left(\mathbf{x}_{i}-\mathbf{y}_{i}\right)\left(\mathbf{x}_{j}-\mathbf{y}_{j}\right)}{r^{2}}\right] \\
& K_{i j}(\mathbf{x}, \mathbf{y})=-\frac{1}{\pi} \frac{\left(\mathbf{x}_{i}-\mathbf{y}_{i}\right)\left(\mathbf{x}_{j}-\mathbf{y}_{j}\right)\left(\mathbf{x}_{k}-\mathbf{y}_{k}\right) n_{k}(\mathbf{y})}{r^{4}}
\end{aligned}
$$

in which $r=|\mathbf{x}-\mathbf{y}|$ is the distance between the source point $\mathbf{x}$ and the field point $\mathbf{y}$, and $\delta_{i j}$ is the Kronecker delta.

In order to approximate the domain integral on the right-hand side of Eq. (5), the Dual Reciprocity idea is used [22]. The time derivative is expanded as

$$
g_{j}(\mathbf{x})=\frac{\partial u_{j}}{\partial t}=\sum_{m=1}^{N} f\left(\mathbf{x}, \mathbf{y}^{m}\right) \alpha_{l}^{m}(t) \delta_{j l}
$$

where $f\left(\mathbf{x}, \mathbf{y}^{m}\right)$ are known functions depending only on geometry, $\boldsymbol{\alpha}^{m}$ are time dependent unknown coefficients and $\mathbf{y}^{m}, m=1,2, \ldots, N$, are $N$ fixed collocation points.

Thus, the domain integral on the right-hand side of Eq. (5) can be written as

$$
\int_{\Omega} u_{j}^{k}(\mathbf{x}, \mathbf{y}) g_{j}(\mathbf{y}) d \Omega_{\mathbf{y}}=\sum_{m=1}^{N} \boldsymbol{\alpha}_{l}^{m} \int_{\Omega} u_{j}^{k}(\mathbf{x}, \mathbf{y}) f\left(\mathbf{x}, \mathbf{y}^{m}\right) \delta_{j l} d \Omega_{y} .
$$

Applying Green's formula to the above equation yields

$$
\begin{aligned}
\int_{\Omega} u_{j}^{k}(\mathbf{x}, \mathbf{y}) g_{j}(\mathbf{y}) d \Omega_{\mathbf{y}}=\sum_{m=1}^{N} & \boldsymbol{\alpha}_{l}^{m}\left[u_{k}^{l}\left(\mathbf{x}, \mathbf{z}^{m}\right)-\int_{\Gamma} K_{k j}(\mathbf{x}, \mathbf{y}) \hat{u}_{j}^{l}\left(\mathbf{y}, \mathbf{z}^{m}\right) d \Gamma_{\mathbf{y}}\right. \\
& \left.+\int_{\Gamma} u_{j}^{k}(\mathbf{x}, \mathbf{y}) \hat{t}_{j}^{l}\left(\mathbf{y}, \mathbf{z}^{m}\right) d \Gamma_{\mathbf{y}}\right]
\end{aligned}
$$

where $\hat{u}_{j}^{l}$ and $\hat{t}_{j}^{l}$ are the particular displacements and tractions defined as

$$
\begin{aligned}
& \hat{u}_{j}^{l}=\frac{1}{15}\left[\frac{4}{3} \delta_{l j}-\frac{\partial r}{\partial x_{l}} \frac{\partial r}{\partial x_{j}}\right] r^{3} \\
& \hat{t}_{j}^{l}=\frac{2}{15}\left[\frac{3}{2} \frac{\partial r}{\partial x_{j}} n_{l}+\frac{3}{2} \frac{\partial r}{\partial x_{l}} n_{j}+\frac{\partial r}{\partial n}\left(\frac{3}{2} \delta_{l j}-\frac{\partial r}{\partial x_{l}} \frac{\partial r}{\partial x_{j}}\right)\right] r^{2}
\end{aligned}
$$


After substituting Eq. (9) into Eq. (5), the BEM is used to discretize the boundary integral equation (5) by dividing the surface $\Gamma$ of the problem into smaller elements as [21]

$$
\begin{aligned}
c(\mathbf{x}) u_{k}(\mathbf{x})-\sum_{n=1}^{N E} \int_{\Gamma_{n}} K_{k j}(\mathbf{x}, \mathbf{y}) u_{j}(\mathbf{y}) d \Gamma_{\mathbf{y}}+\sum_{n=1}^{N E} \int_{\Gamma_{n}} u_{j}^{k}(\mathbf{x}, \mathbf{y}) t_{j}(\mathbf{y}) d \Gamma_{\mathbf{y}}= \\
\sum_{m=1}^{N} \boldsymbol{\alpha}_{l}^{m}\left[\hat{c}_{k j}(\mathbf{x}) u_{j}^{l}\left(\mathbf{x}, \mathbf{z}^{m}\right)-\sum_{n=1}^{N E} \int_{\Gamma_{n}} K_{k j}(\mathbf{x}, \mathbf{y}) \hat{u}_{j}^{l}\left(\mathbf{y}, \mathbf{z}^{m}\right) d \Gamma_{\mathbf{y}}\right. \\
\left.+\sum_{n=1}^{N E} \int_{\Gamma_{n}} u_{j}^{k}(\mathbf{x}, \mathbf{y}) \hat{t}_{j}^{l}\left(\mathbf{y}, \mathbf{z}^{m}\right) d \Gamma_{\mathbf{y}}\right]
\end{aligned}
$$

where $N E$ is the number of elements.

When Eq. (11) is applied at all collocation points, the set of equations produced can be written in matrix-vector form as

$$
H U-G T=(H \hat{U}-G \hat{T}) \alpha
$$

where $U$ and $T$ are velocity and traction vectors at each nodal point, respectively. $H$ and $\hat{U}$ are $(2 N \times 2 N), G$ is $(2 N \times 3 N)$ and $\hat{T}$ is $(3 N \times 2 N)$ matrices in which $N$ is the number of boundary nodes. $\hat{U}$ and $\hat{T}$ are constructed by taking $\hat{u}_{j}^{l}$ and $\hat{t}_{j}^{l}$ as columns.

The $\boldsymbol{\alpha}$ vector can be obtained by inverting Eq. (7) so that Eq. (12) can be written as

$$
H U-G T=(H \hat{U}-G \hat{T}) F^{-1} \dot{U} .
$$

in which $F$ is a $(2 N \times 2 N)$ matrix containing the coordinate functions, $f\left(\mathbf{x}, \mathbf{y}^{m}\right)$, as columns evaluated at each boundary point.

The time derivative is approximated by a central difference scheme

$$
H U^{(m+1)}-G T^{(m+1)}=(H \hat{U}-G \hat{T}) F^{-1} \frac{U^{(m+1)}-U^{(m-1)}}{2 \Delta t}
$$

In order to calculate $U^{(2)}$ (when $m=1$ ), two initial conditions are required. Thus, the equation is solved first using a forward difference scheme to calculate $U^{(1)}$ by setting the values of $U^{(0)}$. Then, Eq. (14) is solved step by step for $m \geq 1$.

Rearranging Eq. (14) yields

$$
\tilde{H} U^{m+1}-G T^{m+1}=d .
$$

where $S=(H \hat{U}-G \hat{T}) F^{-1}, \tilde{H}=\left(H-\frac{S}{2 \Delta t}\right)$ and $d=-\frac{S}{2 \Delta t} U^{(m-1)}$. 
Then, the boundary conditions are inserted into Eq. (15). When the boundary conditions are no-slip the corresponding columns of $\tilde{H}$ and $G$ are interchanged so that the known values are transposed to the right-hand side and the unknown values are collected on the left-hand side of the equation. On the other hand, when the boundary conditions are slip, the columns of the matrices are arranged using the slip condition

$$
u_{i}^{f}-U_{i}^{w}=L s t_{i}
$$

as follows

$$
\begin{aligned}
& \tilde{H}\left(L s t_{i}+U_{i}^{w}\right)-G t_{i}=d \\
& (L s \tilde{H}-G) t_{i}=d-\tilde{H} U_{i}^{w}
\end{aligned}
$$

Finally, the system can be written in the form

$$
A x=b
$$

and solved for the unknowns $x=t_{i}$, then $u_{i}$ 's are calculated using Eq. (16).

\section{Results and Discussion}

Quadratic boundary elements and linear radial basis functions, $f=1+r$, are used in the DRM formulation. Solutions are presented at steady-state in which the pre-assigned tolerance is taken as

$$
\max _{m=1, N+L}\left|u^{m+1}-u^{m}\right| \leq 10^{-7}
$$

where $N$ and $L$ are the number of boundary and internal nodes, respectively.

\subsection{Flow in a horizontal channel}

The simple problem of flow in a horizontal channel shown in Fig (1) is investigated for linear and nonlinear slip boundary conditions. The linear slip boundary condition is considered for two slip length values, $L s=0.25$ and 0.5 , while the channel length varies from $L=2$ to 10 . The nonlinear slip boundary condition is applied by taking $n=1$, $0<\beta \leq 0.4$ and $L=2$.

The analytical solution of the linear and nonlinear slip problem in dimensional form is given respectively as [15]

$$
\begin{gathered}
u_{1}=\frac{h^{2}}{2 \mu} \frac{\Delta P}{L}\left[1-\left(\frac{y}{h}\right)^{2}\right]+\frac{h}{\mu} \frac{\Delta P}{L} L s \\
u_{1}=\frac{h^{2}}{2 \mu} \frac{\Delta P}{L}\left[1-\left(\frac{y}{h}\right)^{2}\right]+\frac{h}{\mu} \frac{\Delta P}{L} \frac{b_{0}}{\sqrt{(1-2 \beta)^{n}}}
\end{gathered}
$$


where $L$ is the channel length, $2 h=2$ is the channel height, $\Delta P=P_{0}-P_{L}=1$ is the imposed pressure difference, $\mu=1$ is the dynamic viscosity and $L s$ is the slip length. The velocity reduces to no-slip when the slip length is dropped to zero.

The boundary conditions are specified in Fig. (1). The value of $t_{2}$ at the horizontal walls is given by the pressure $p$, which varies linearly between its given values at the inlet and outlet.

Fig. (2) shows the results for $L s=0.25$ and 0.5 when the channel length is $L=2$ and 10 . For $L=2,50$ boundary elements are used in the discretization of the boundary. Since the channel is short, the horizontal boundaries are divided into 15 elements while the vertical boundaries are divided into 10 elements. The time step is taken as $\Delta t=0.1$. As the channel length increases to 10, more boundary elements are needed. Thus, 70 boundary elements are used in the discretization. The horizontal boundaries are divided into 25 elements while the same number of elements are used for the vertical boundaries. It is also observed that a smaller time step is needed with an increase in the channel length. Thus, the time step $\Delta t$ is reduced to 0.05 . Note that, for the values adopted, the velocity $u_{1}$, at the horizontal

boundaries $(y=-h, h)$ can be calculated as $u_{1}=\frac{L s}{L}$. It is clear from Fig. (2) that the numerical values are in excellent agreement with the analytical ones

Fig. (3) presents the transient behaviour of the flow for various time levels when $L s=0.25$ and 0.5 with $L=10$, respectively. It can be seen that the flow becomes steady in a short period of time.

Table (1) presents the maximum and minimum values of the computed velocity with linear slip at different time levels for $L s=0.25$ and 0.5 when the length of the channel is $L=10$. These values occur at the channel centre and at the wall, respectively. One can see that convergence is obtained after 50 iterations. The results are in very good agreement with the exact solution at steady-state. One can see from Table (1) that for $L s=0.25$ and 0.5 , the errors for the maximum and minimum velocities are $10^{-7}$ and $10^{-6}$, respectively.

Fig. (4) shows the velocity profiles for various nonlinear slip length values when the channel length is $L=2$ and $b_{0}=0.5$. Fig. (5) shows a comparison of these velocity profiles with the analytical solution. Tables (2) and (3) present the maximum and minimum values of the computed and exact velocities for several nonlinear slip values $0<\beta \leq 0.4$ when the channel length is $L=2$ and $b_{0}=0.5$. Again, these values occur at the channel centre and at the wall, respectively. The BEM solutions are in good agreement with the above steady-state analytical solution.

\subsection{Backward-facing step}

As a second test problem, a backward-facing step flow is considered for several slip length values, Ls, from 0 to 0.75. The boundary conditions are given in Fig. (6). Linear slip boundary conditions are inserted at the bottom and top walls of the channel, as well as downstream of the step. At the inlet of the channel a parabolic velocity profile is imposed as boundary condition, and at the outlet a developed flow is considered. Discretization of the channel is more refined from $x=0$ to $x=4$. Overall, 224 boundary elements are used with time step $\Delta t=0.001$. 
Fig. (7) presents the flow downstream of the step up to the outlet for $\Delta t=0.005,0.003,0.001$ and 0.0008. The mentioned convergence criteria is reached after 122,80, 50 and 30 time steps, respectively.

The transient behaviour of the flow is shown in Fig. (8) at different sections of the channel from downstream of the step up to the outlet for slip length $L s=0.5$. It is observed that the flow reaches steady-state in a short time. Fig. (9) presents the velocity profiles at the midplane and outlet of the channel for different time steps. It can be seen that at steady-state, the flow is fully developed and preserves its parabolic shape up to the outlet.

Fig. (10) shows the horizontal velocity profiles at different sections of the channel from inlet to centerline for no-slip and several slip length values at steady-state. It is observed that at the inlet, the parabolic shape is preserved and it is shifted to the right depending on the slip length value. It is also observed that after the step, the parabolic behaviour is distorted up to the centerline. Furthermore, the effect of the slip condition is more pronounced at the upper wall of the channel due to the step being placed at its left bottom corner.

\subsection{Lid-driven cavity flow}

The lid-driven cavity flow is considered as the third problem. Boundary conditions can be seen in Fig. (11). The upper wall of the cavity is moving to the right with constant velocity, while the lower wall is either moving in the same $(S=1)$ or in the opposite $(S=-1)$ directions. Linear slip boundary condition is applied on the vertical and horizontal walls of the cavity.

In order to validate the formulation, different boundary conditions are initially considered. Fig. (12a) shows the solution when both the left and right walls of the cavity move up with velocity $u_{2}=1$, while Fig. (12b) shows the solution when the left wall moves down with velocity $u_{2}=-1$ and the right wall moves up with velocity $u_{2}=1$, with the bottom and top walls are stationary. These solutions are in good agreement with those presented in Fig. 4-(a),(c) of Kelmanson and Lonsdale [23]. Fig. (12c) presents the solution when the upper and lower walls move in the same direction, $S=1$ (in this case the top wall of the cavity moves to the right with velocity $u_{1}=2$ ), while Fig. (12d) presents the solution when the upper and lower walls move in opposite directions, $S=-1$, with the vertical walls stationary. These solutions are in good agreement with the ones presented in Fig. 2-(d),(f) of Gaskell et al. [24].

The effect of the number of boundary elements is tested in Fig. (13) for the case $S=1$. The boundary of the cavity is discretized into 120,160,320 and 360 elements, respectively. The time step is taken as $\Delta t=0.005$. It was noted that more boundary elements are needed with the same time step value when the horizontal walls are moving in the opposite directions, i.e. $S=-1$.

Flow in the cavity for the cases $S=1$ and $S=-1$ with $0 \leq L s \leq 1$ are presented in Figs. (14) and (15), respectively. When the horizontal walls of the cavity move in the same direction, $S=1$, the velocity field has two main circulations. The upper one is in the clockwise direction and the lower one is in the counter clockwise direction. It is observed that as the slip length value increases to 1 , the main circulation cells move through the corners of the cavity. When the walls of the cavity are moving in opposite directions, $S=-1$, there is one main circulation cell which is in the clockwise direction and its center has an 
elliptical shape. As the slip length value increases the elliptical shape in the center of the cavity becomes circular and the streamlines become parallel to the walls.

In Fig. (16), the effect of the slip length value $(0 \leq L s \leq 1)$ on the vertical and horizontal velocity profiles along the centerline of the cavity are presented for both cases. It is observed that the magnitude of the vertical and horizontal velocity decreases with an increase in slip

length value. For the case $S=-1$ the magnitude of the vertical velocity is greater than the one for $S=1$, and the decrease with respect to the slip length value is more pronounced.

\section{Conclusions}

In this study, transient slip Stokes flow in different geometries is numerically investigated using DRBEM. The time derivative is approximated by the FDM. Linear and nonlinear slip boundary conditions are applied. For the channel flow problem, comparisons are made with analytical solutions for both linear and nonlinear slip boundary conditions. For the step flow problem, it is observed that the flow is fully developed at the mid-plane and has the same parabolic behaviour up to the outlet. In the cavity problem, it is observed that more boundary elements are needed when the walls of the cavity are moving in opposite directions, but the time step value can be kept the same. It is also observed that the effect of the slip length value on the velocity profiles is more pronounced in this case. The time step value is selected depending on the geometry of the problem. Due to the implicit nature of the time integration scheme, quite large time steps can be taken.

\section{References}

[1] Nguyen N. and Wereley S. (2006), Fundamentals and Applications of Microfluidics. Artech House, Norwood.

[2] Granick S. (1991), Motions and relaxations of confined liquids. Science, Vol. 253, pp 1374-1379.

[3] Lamb H. (1932), Hydrodynamics. Cambridge University Press, Cambridge.

[4] Neto C., Craig V. and Williams D.R.M. (2003), Evidence of shear-dependent boundary slip in Newtonian liquids. The European Physical Journal E: Soft Matter and Biological Physics, Vol. 12, pp 71-74.

[5] Neto C., Evans D.R., Bonaccurso E., Butt H. and Craig V. (2005), Boundary slip in Newtonian liquids: A review of experimental studies. Reports on Progress in Physics, Vol. 68, pp 2859-2897.

[6] Matthews M.T. and Hill J.M. (2007), Newtonian flow with nonlinear Navier boundary conditions. Acta Mechanica, Vol. 191, pp 195-217. 
[7] Thompson P.A. and Troian S.M. (1997), A general boundary condition for liquid flow at solid surfaces, Nature, Vol. 389, pp 360-362.

[8] Kuo L.S. and Chen P.H. (2009), A unified approach for nonslip and slip boundary conditions in the lattice Boltzmann method. Computers and Fluids, Vol 38, pp 883-887.

[9] Power H., Soavi J., Kantachuvesiri P. and Nieto C. (2015), The effect of Thompson and Troian's nonlinear slip condition on Couette flows between concentric rotating cylinders. Z. Angew Math. Phys., Vol 66, pp 2703-2718.

[10] Luo H. and Pozrikidis C. (2008), Effect of surface slip on Stokes flow past a spherical particle in infinite fluid and near a plane wall. Journal of Engineering Mathematics, Vol. 62, pp 1-21.

[11] Ding J. and Ye W. (2004), A fast integral approach for drag force calculation due to oscillatory slip Stokes flows. International Journal for Numerical Methods in Engineering, Vol. 60, pp 1535-1567.

[12] Frangi A., Spinola G. and Vigna B. (2006), On the evaluation of damping in MEMS in the slip flow regime. International Journal for Numerical Methods in Engineering, Vol. 68, pp 1031-1051.

[13] Navier C.L.M.H. (1823), Mémoire sur les lois du mouvement des fluides. Mémoire de l'Académie Royale des Sciences de l'Institut de France, VI, pp 389-440.

[14] Basset A.B. (1961), A Treatise on Hydrodynamics. Dover, New York.

[15] Nieto C., Giraldo M. and Power H. (2011), Boundary integral equation approach for Stokes slip flow in rotating mixers. Discrete and Continuous Dynamical Systems, Series B, Vol. 15, pp 1019-1044.

[16] Nieto C., Power H. and Giraldo M. (2013), Boundary elements solution of Stokes flow between curved surfaces with nonlinear slip boundary condition. Numerical Methods for Partial Differential Equations, Vol. 29, pp 757-777.

[17] Myong R.S., Reese J.M., Barber R.W., Emerson, D.R. (2005), Velocity slip in microscale cylindrical Couette flow: the Langmuir model. Phys. Fluids, Vol. 17, 087105-1087105-11.

[18] Zhou P., Cheng-wei W., Guo-jun M. (2007), Nonlinear boundary slip of fluid flowing over solid surface. J. Cent. South Univ. Technol. Vol. 14, pp 3033.

[19] Darbandi M., Mahboubi Fouladi H., Zakyani M., Schneider G.E. (2013), Numerical simulation of slip flow through microchannels; described in the curvilinear coordinate system. In: Proceedings of the International Conference on Mechanical Engineering and Mechatronics, Toronto, Ontario, Canada.

[20] Power H. and Partridge P. W. (1993), Dual reciprocity boundary element method for time-dependent Stokes flows. International Journal for Numerical Methods in Heat and Fluid Flow, Vol. 3, pp 145-155. 
[21] Power H. and Wrobel L. C. (1995), Boundary Integral Methods in Fluid Mechanics. Computational Mechanics Publications, Southampton.

[22] Partridge P. W., Brebbia C. A. and Wrobel L. C. (1992), The Dual Reciprocity Boundary Element Method. Computational Mechanics Publications, Southampton and Elsevier, London.

[23] Kelmanson M. A., Lonsdale B. (1996), Eddy genesis in the double-lid-driven cavity. Quarterly Journal of Mechanics and Applied Mathematics, Vol. 49, pp 635-655.

[24] Gaskell P. H., Savage M. D., Summers J. L., Thompson H.M. (1998), Stokes flow in closed, rectangular domains. Applied Mathematical Modelling, Vol. 22, pp 727-743. 


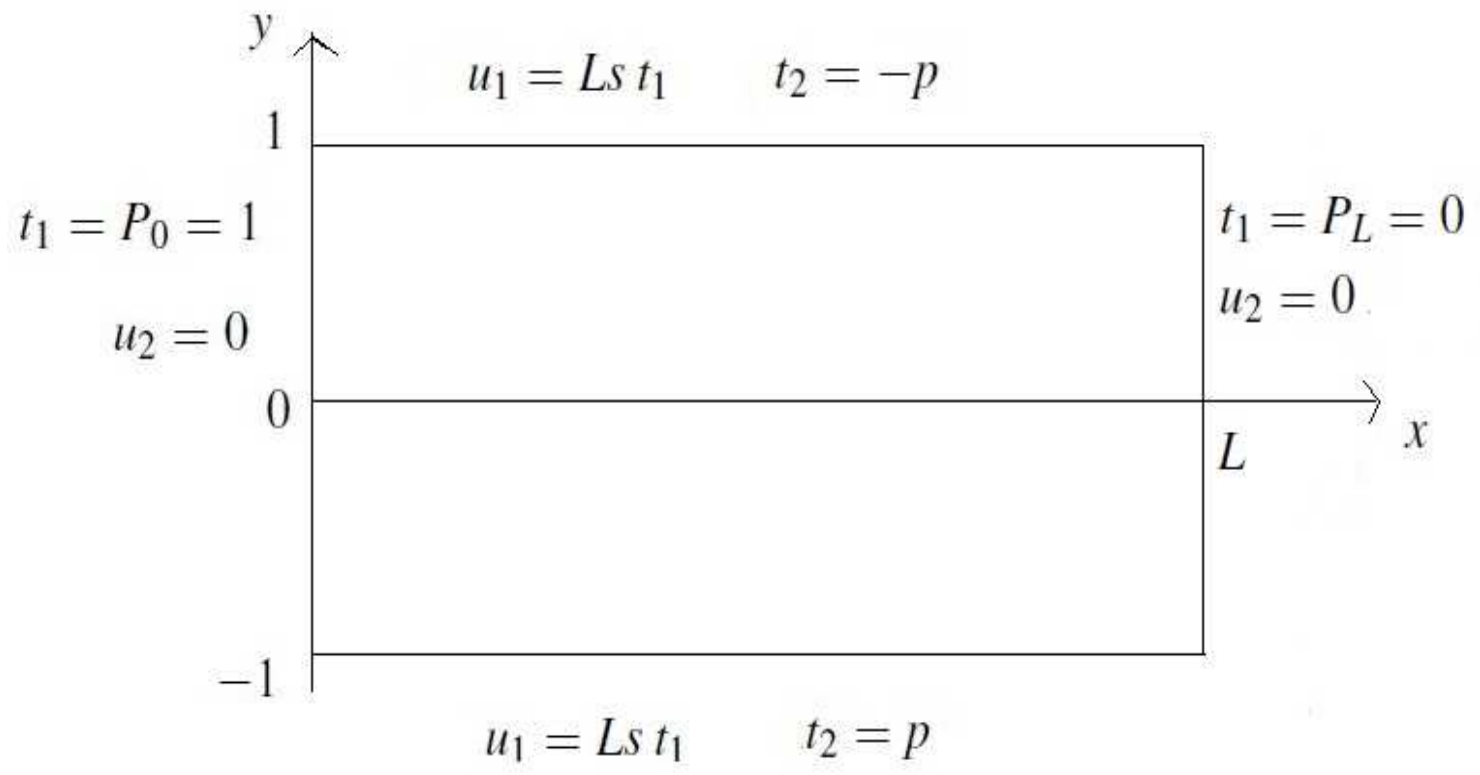

Figure 1: Geometry of Problem 1 

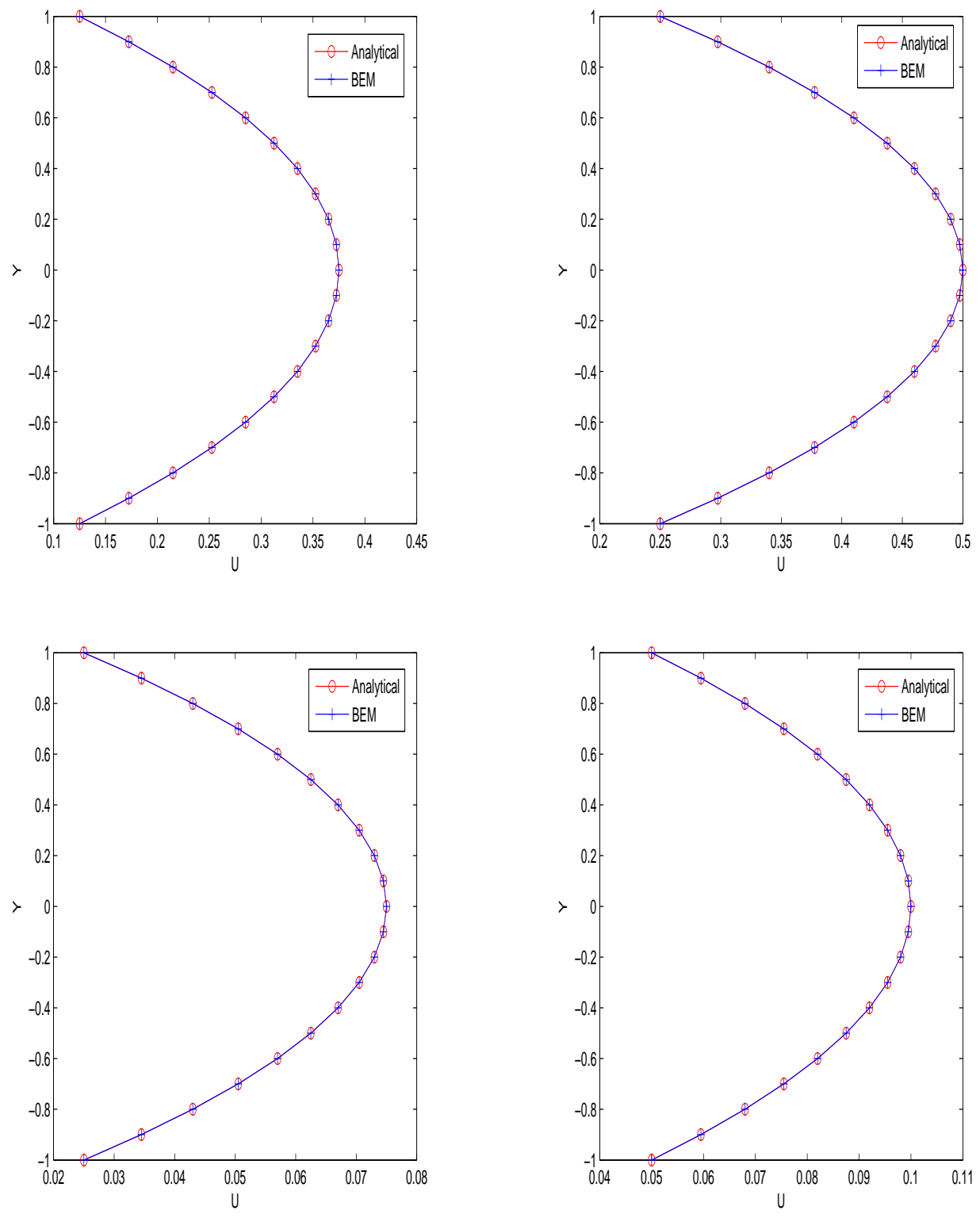

$$
L s=0.25
$$

$$
L s=0.5
$$

Figure 2: Comparison of the velocity profiles at the centerline of the channel when $L=2$ (first row) and $L=10$ (second row) 

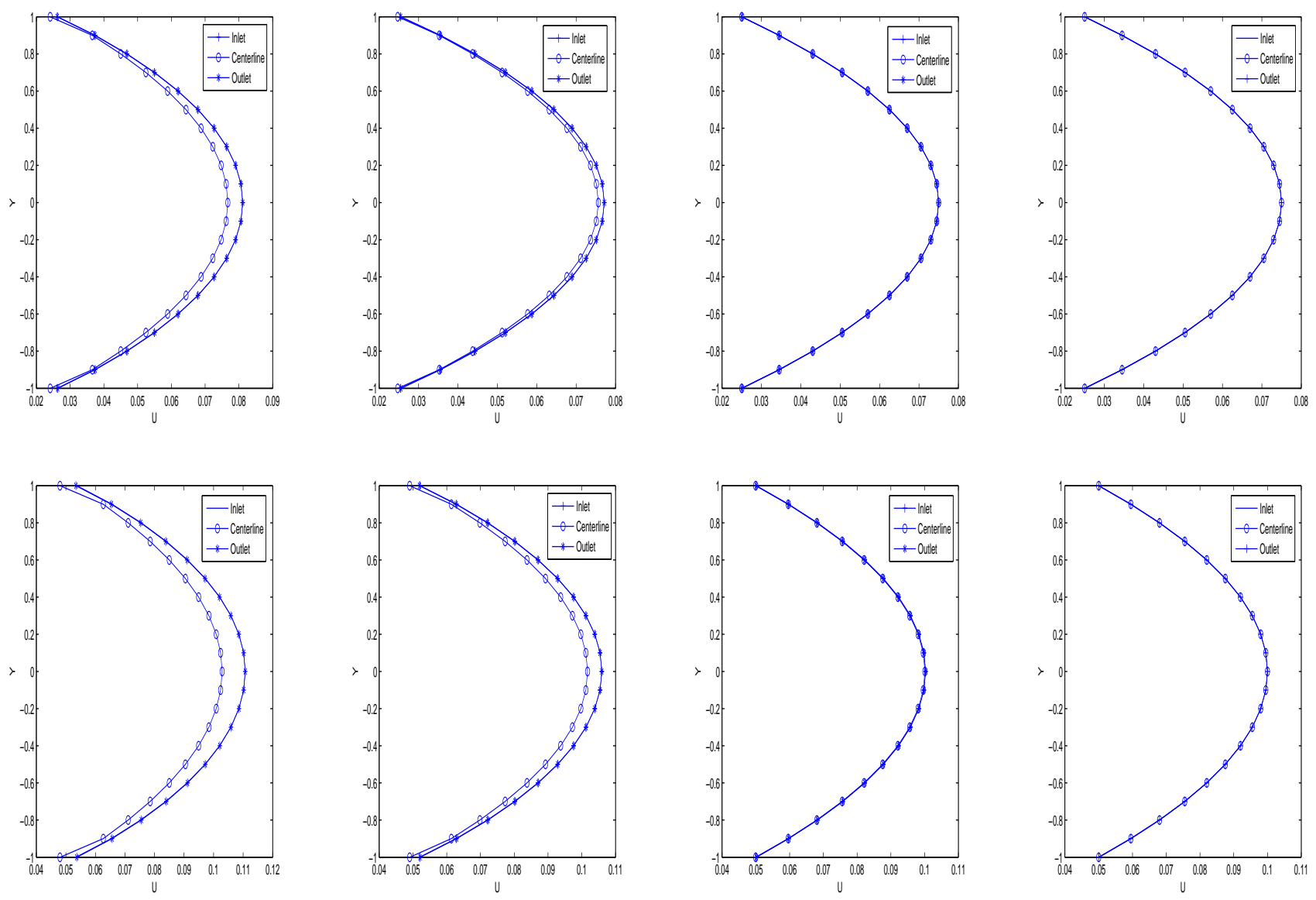

\section{Time Step $=3$}

Time Step $=5$

Time Step $=15$

Time Step $=50$

Figure 3: Transient behaviour of velocity profiles at the inlet, centerline and outlet of the channel with $L=10$ for $L s=0.25$ (first row) and $L s=0.5$ (second row). 
Table 1: Maximum and minimum values of computed velocity at different time levels for $L=10$

\begin{tabular}{lllll}
\hline Time Step & $\begin{array}{l}\text { Ls }=0.25 \\
\text { Max Value }\end{array}$ & Min Value & Max Value & Min Value \\
\hline 3 & .0811364 & .0240827 & .1107291 & .0479862 \\
5 & .0771529 & .0247043 & .1059491 & .0490092 \\
15 & .0750109 & .0249984 & .1002880 & .0499520 \\
50 & .0750000 & .0249999 & .1000000 & .0499999 \\
\hline Exact Solution & .0750000 & .0250000 & .1000000 & .0500000 \\
\hline Error & .0000000 & .0000001 & .0000000 & .0000001 \\
\hline
\end{tabular}




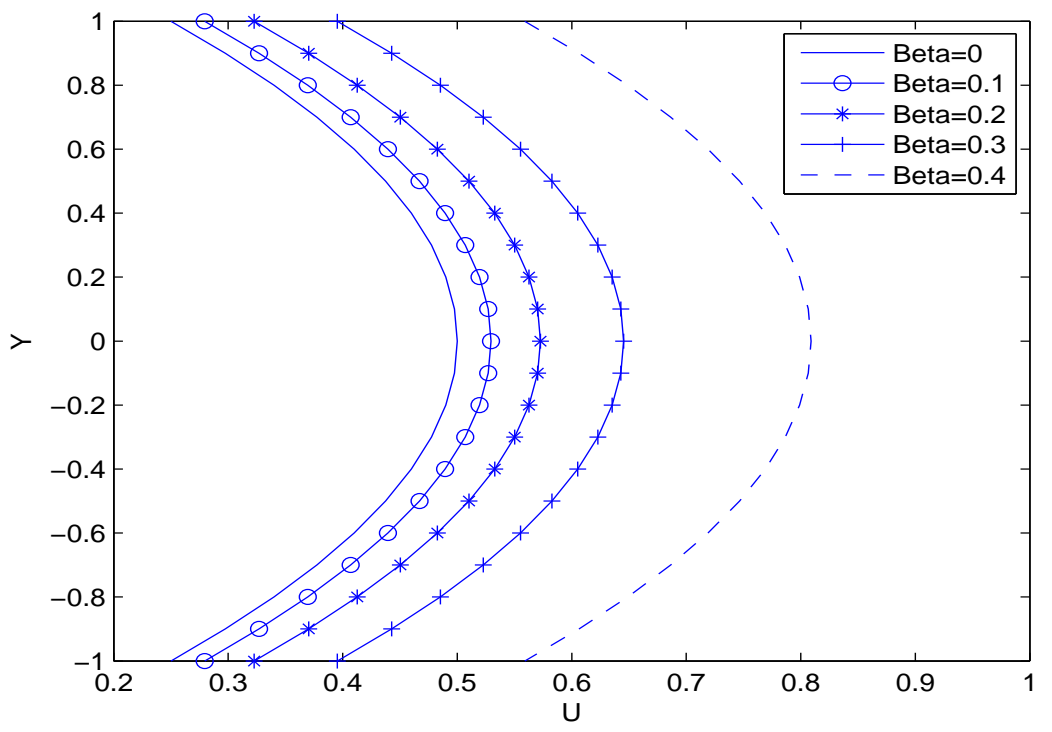

Figure 4: Velocity profiles at the centerline for several nonlinear slip length values when $L=2$ and $b_{0}=0.5$ 

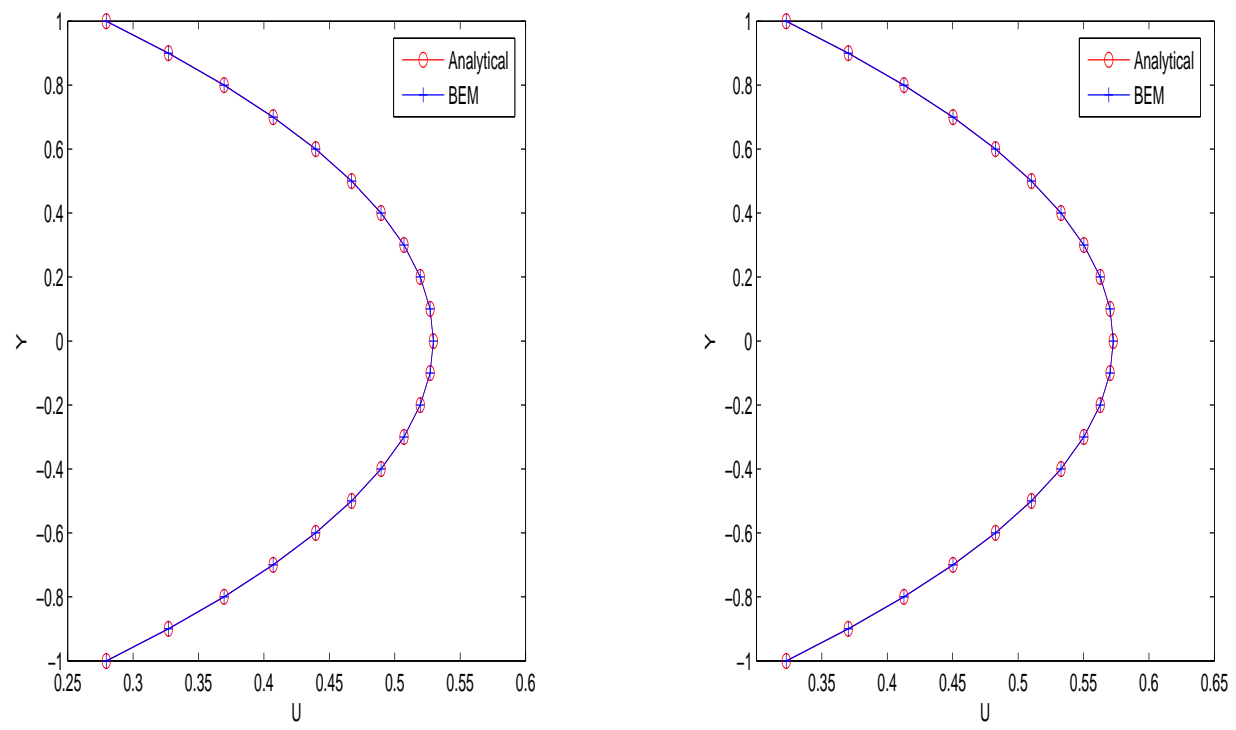

$$
\beta=0.1
$$
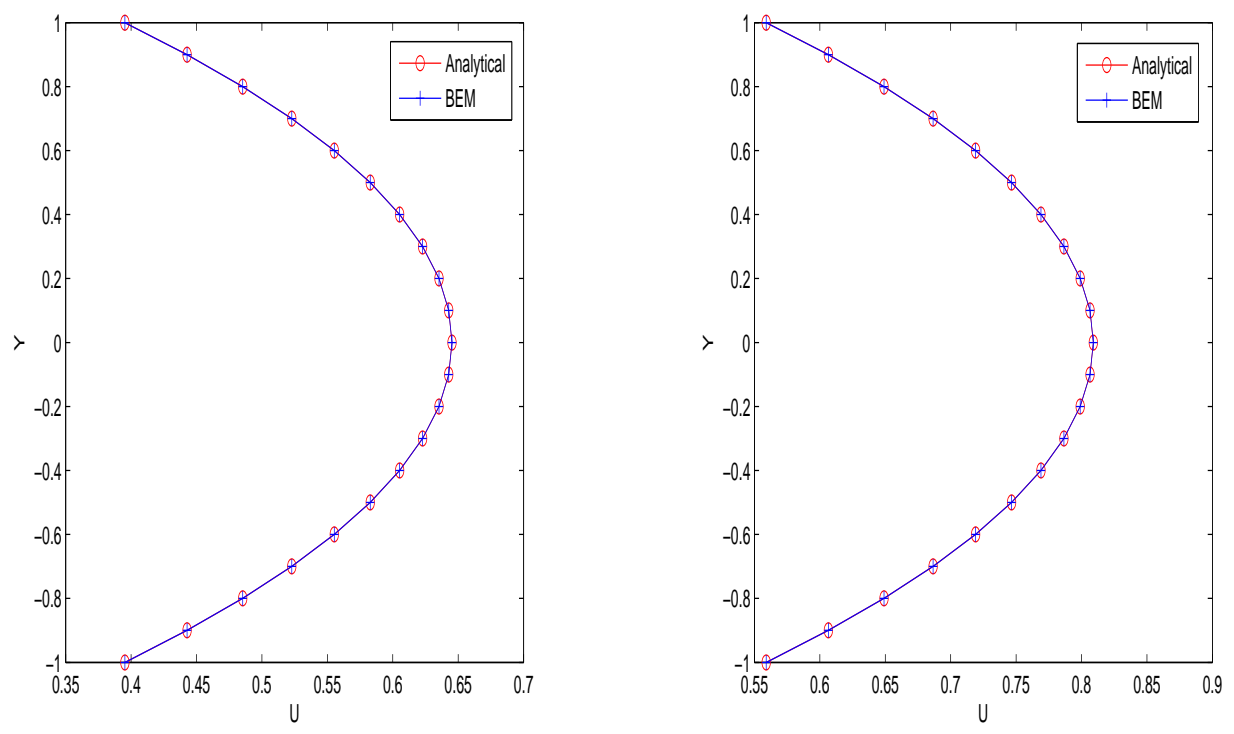

$$
\beta=0.3
$$

$$
\beta=0.4
$$

Figure 5: Comparison of the velocity profiles at the centerline of the channel for several non-linear slip length when $L=2$ and $b_{0}=0.5$ 
Table 2: Maximum values of computed and exact velocity and error at steady-state for several nonlinear slip length value when $L=2$ and $b_{0}=0.5$

\begin{tabular}{llll}
\hline$\beta$ & Computed Solution & Exact Solution & Error \\
\hline 0.1 & .5295085 & .5295084 & .0000001 \\
0.2 & .5727487 & .5727486 & .0000001 \\
0.3 & .6452848 & .6452847 & .0000001 \\
0.4 & .8090171 & .8090169 & .0000002 \\
\hline
\end{tabular}

Table 3: Minimum values of computed and exact velocity and error at steady-state for several nonlinear slip length value when $L=2$ and $b_{0}=0.5$

\begin{tabular}{llll}
\hline$\beta$ & Computed Solution & Exact Solution & Error \\
\hline 0.1 & .2795084 & .2795084 & .0000000 \\
0.2 & .3227485 & .3227486 & .0000001 \\
0.3 & .3952846 & .3952847 & .0000001 \\
0.4 & .5590169 & .5590169 & .0000000 \\
\hline
\end{tabular}




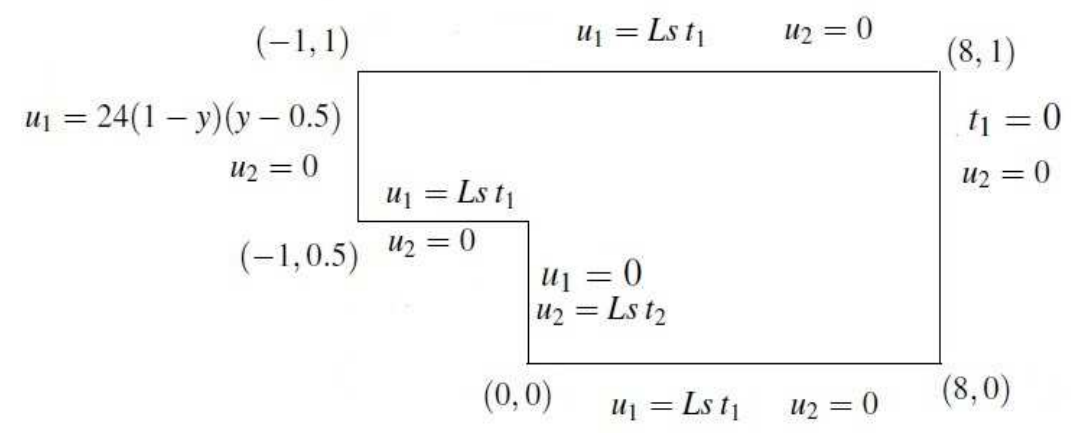

Figure 6: Geometry of Problem 2 

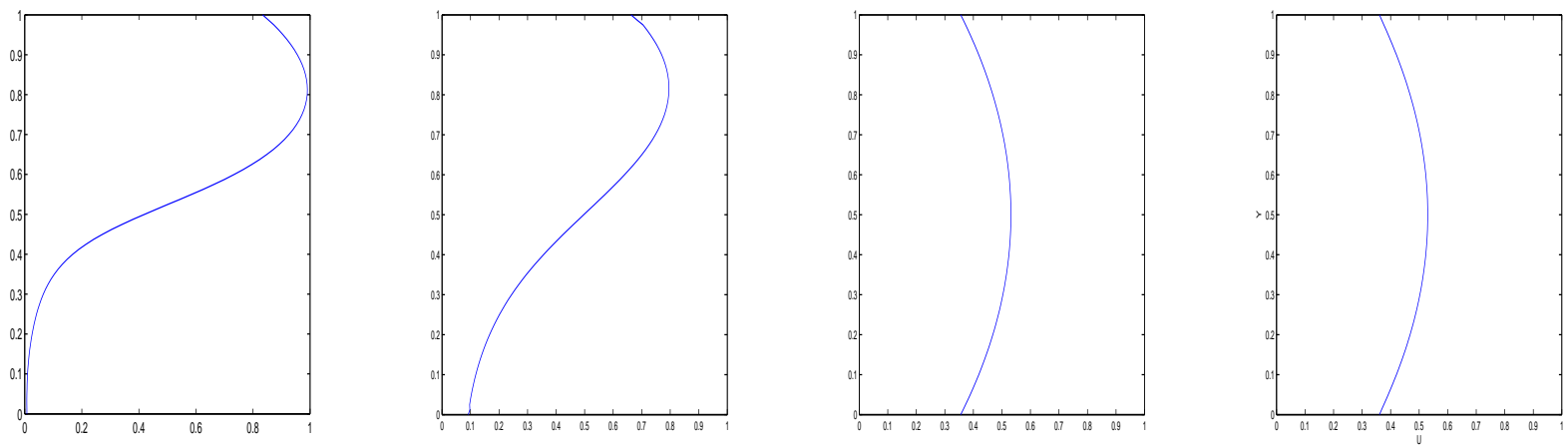

$\Delta t=0.005$
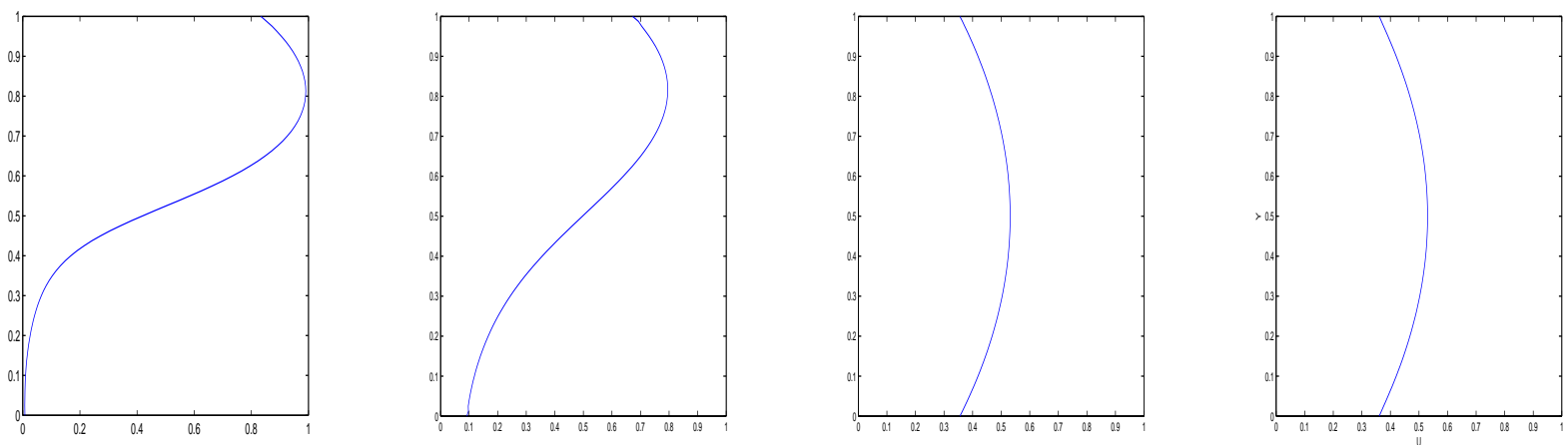

$\Delta t=0.003$
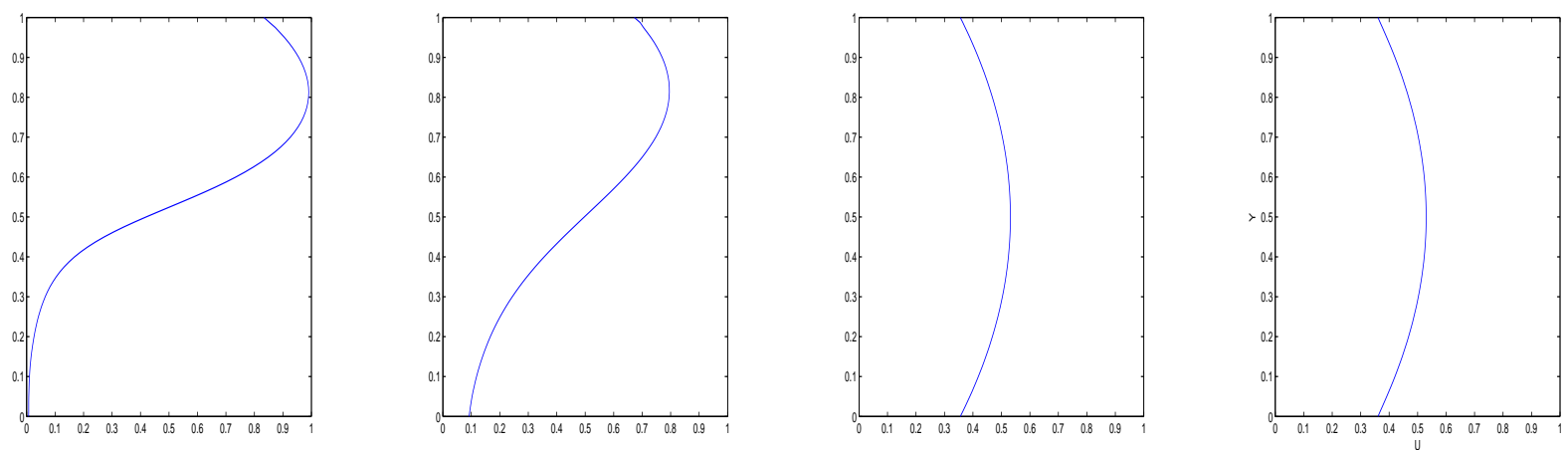

$\Delta t=0.001$
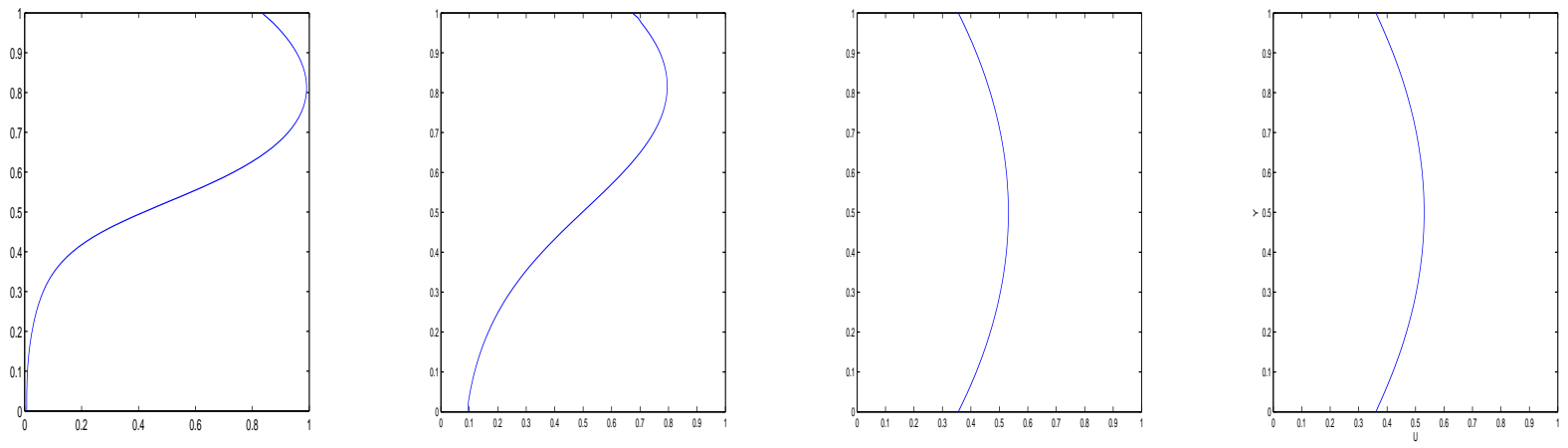

$\Delta t=0.0008$

Figure 7: Steady-state solution for different time-step values for $L s=0.5$. 

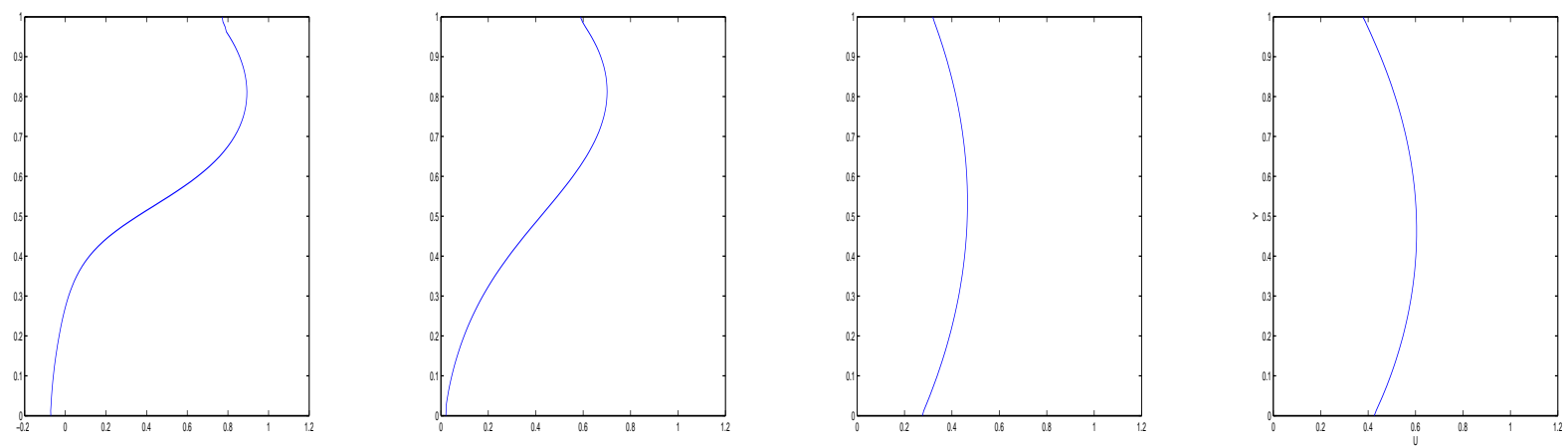

Time Step=6
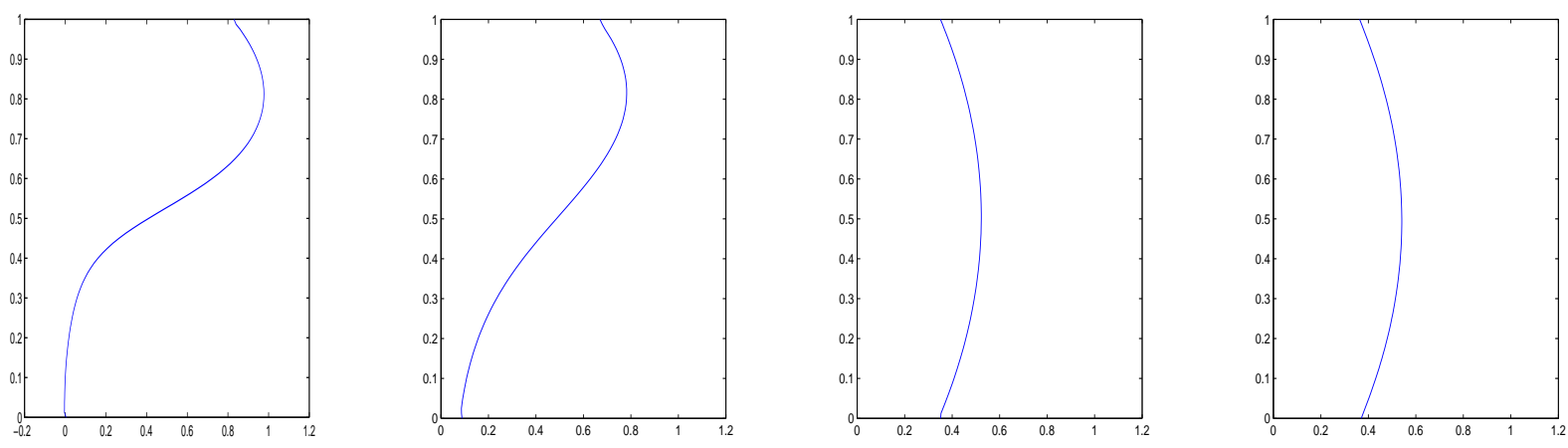

Time Step $=10$
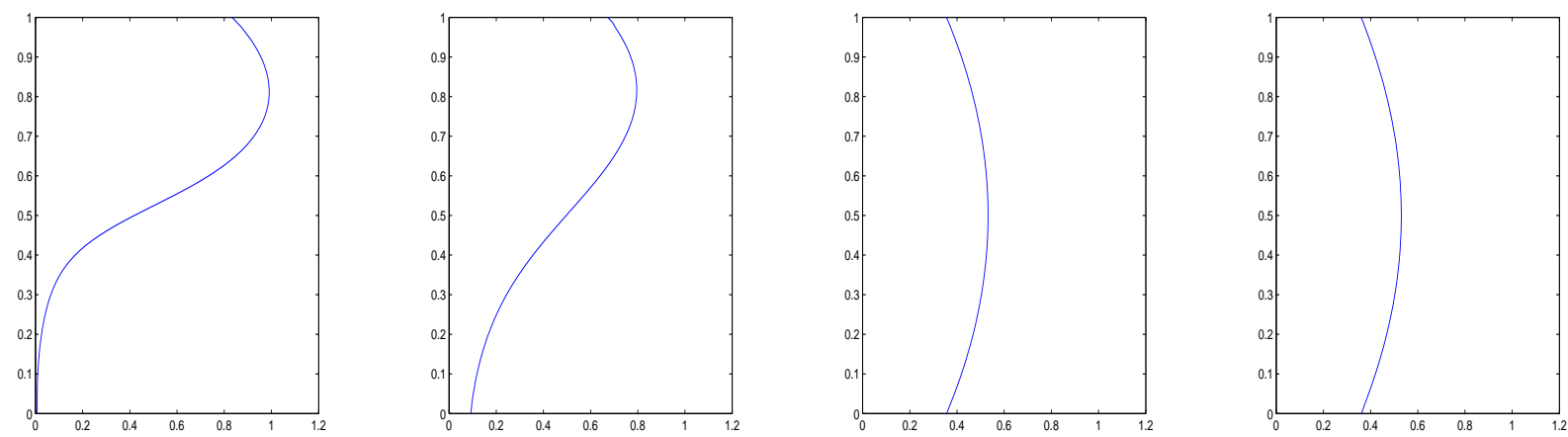

Time Step $=20$
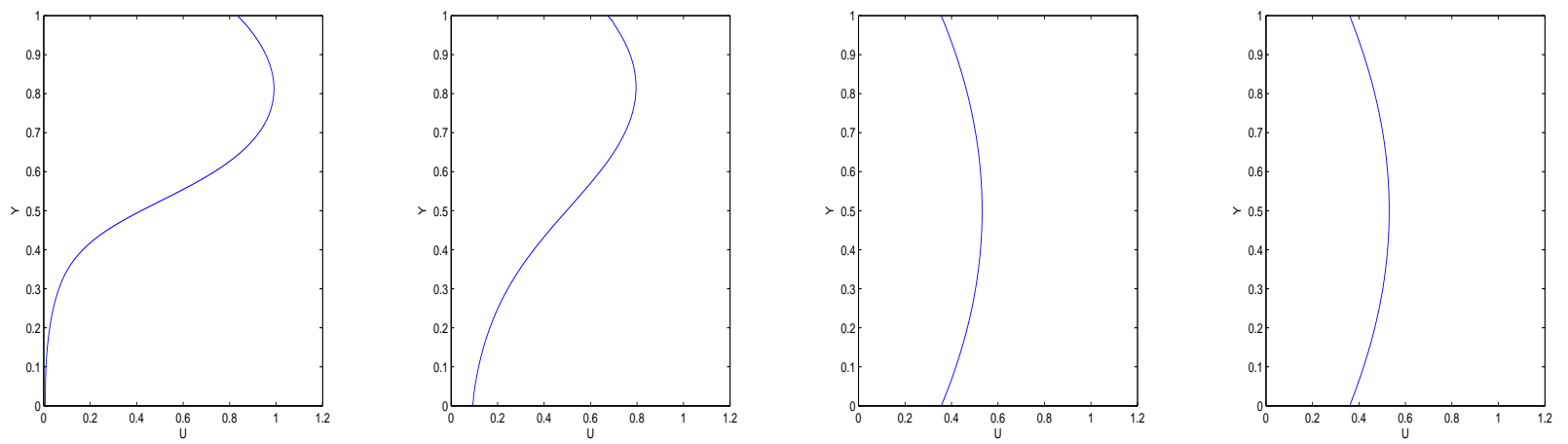

Time Step $=30$

Figure 8: Transient velocity profiles at $x=0.171, x=0.4, x=4$ and $x=8$, respectively, $L s=0.5$. 


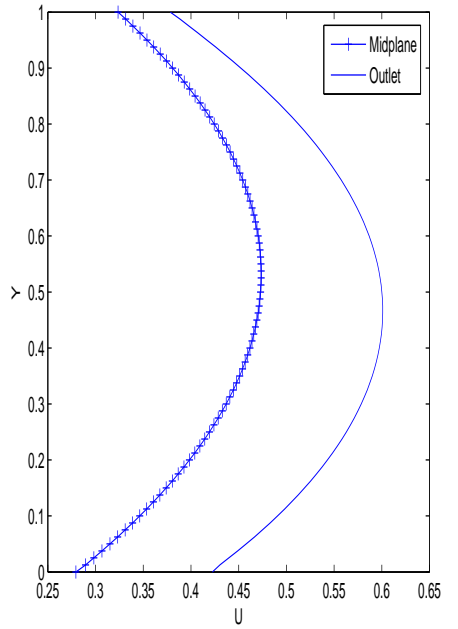

Time Step $=6$

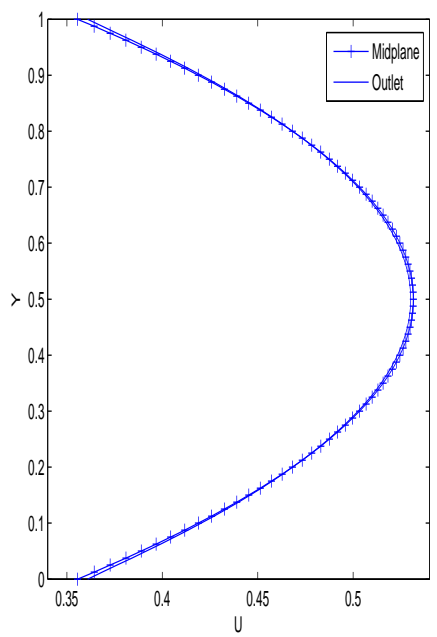

Time Step $=20$

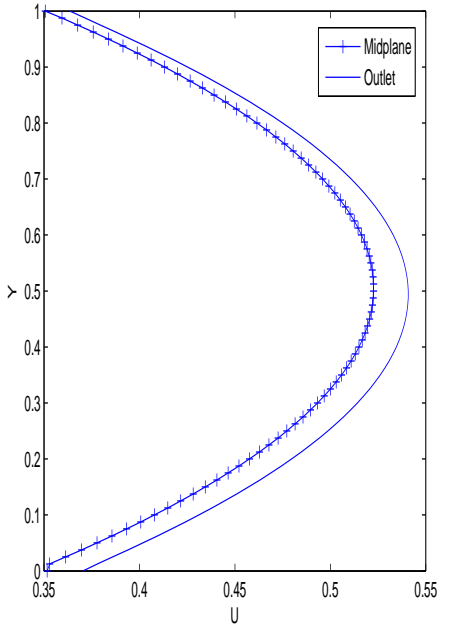

Time Step $=10$

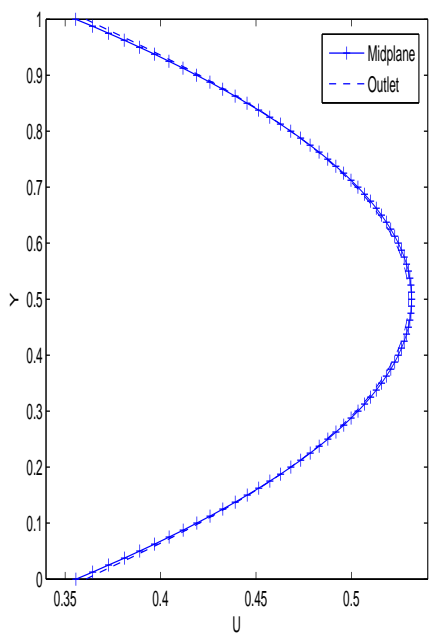

Time Step $=50$

Figure 9: Comparison of transient behaviour of the flow at the midplane and outlet for $L s=0.5$. 

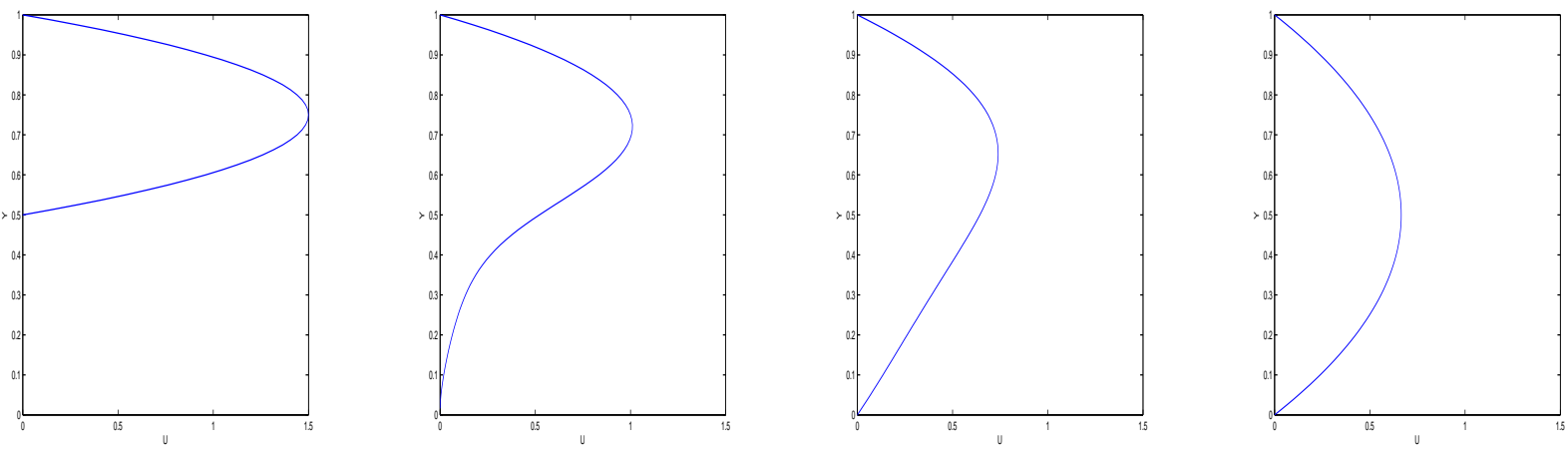

$L s=0$ (no-slip)
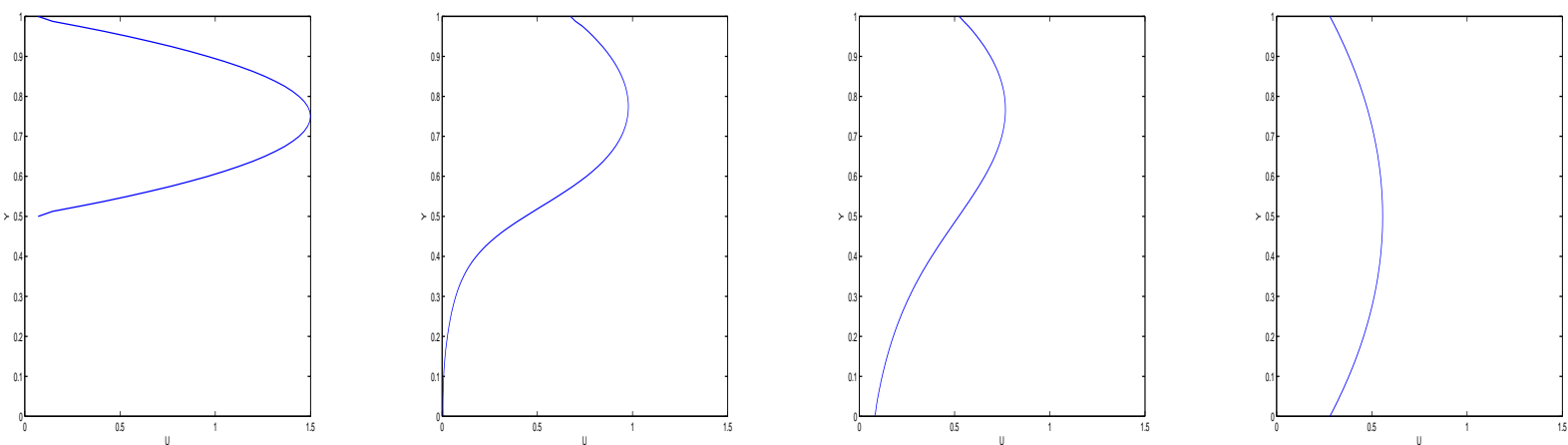

$L s=0.25$
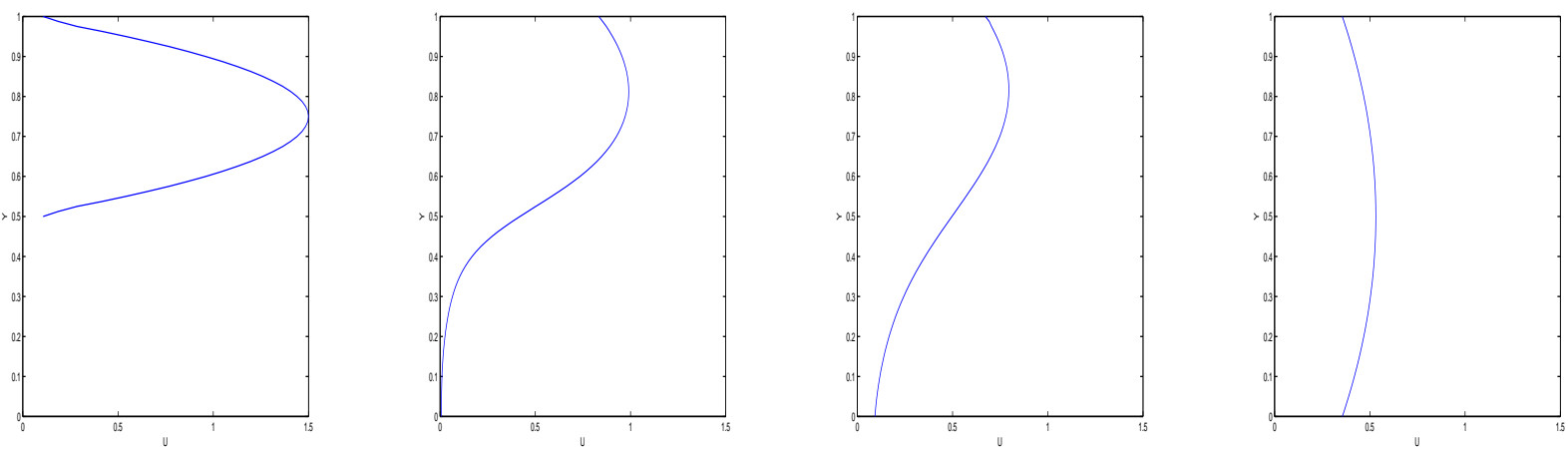

$L s=0.5$
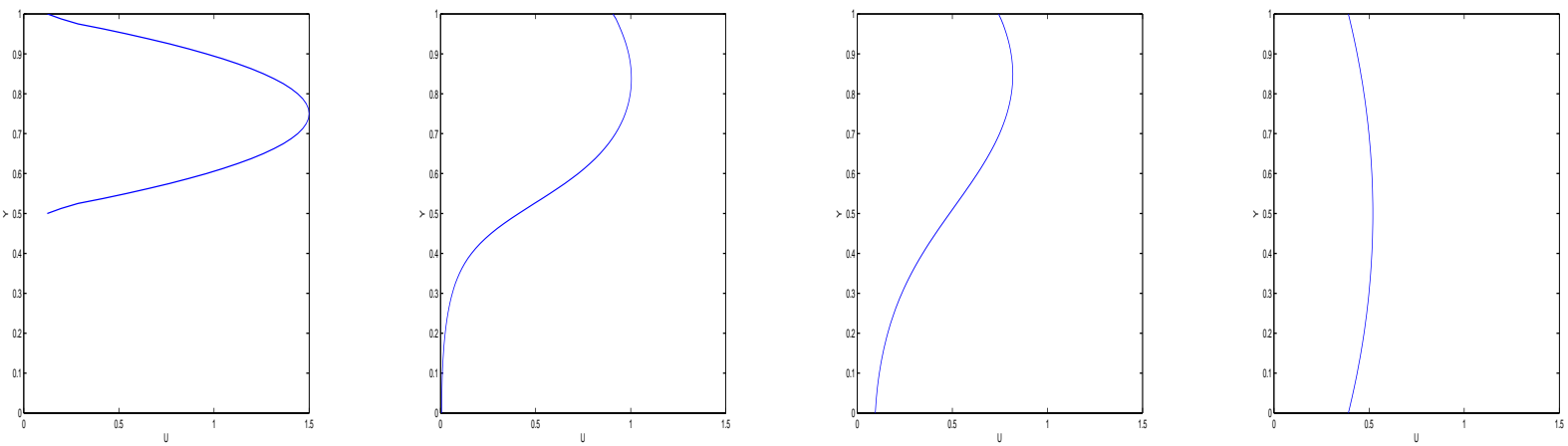

$$
L s=0.75
$$

Figure 10: Velocity profiles at $x=-1, x=0.171, x=0.4$ and $x=4$, respectively, for no-slip and various linear slip length values. 


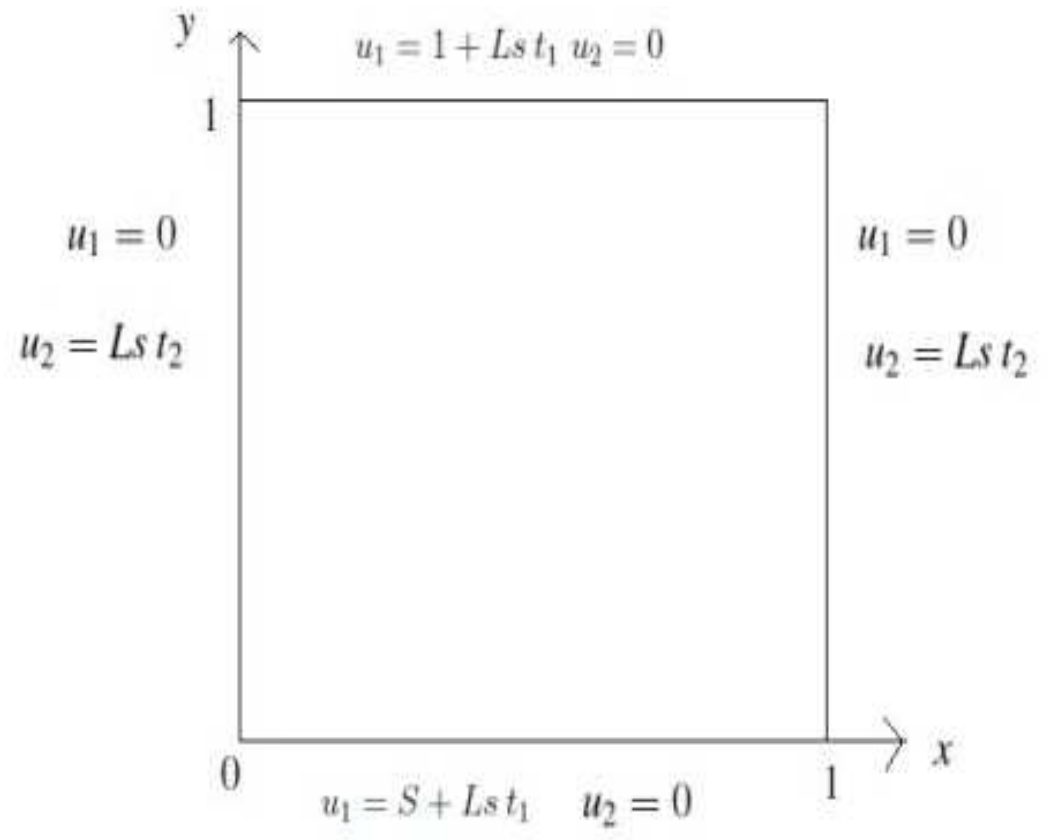

Figure 11: Geometry of Problem 3 


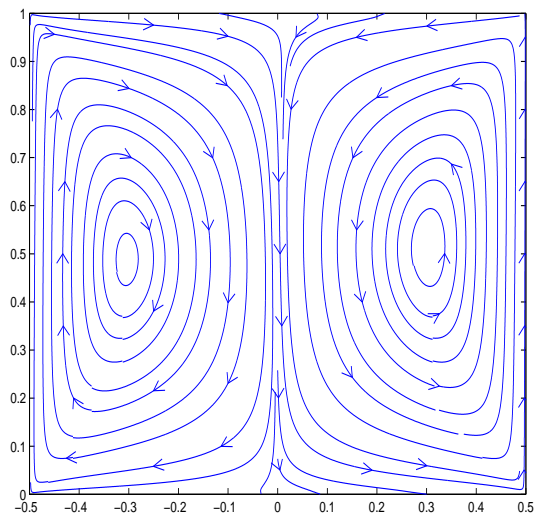

(a)

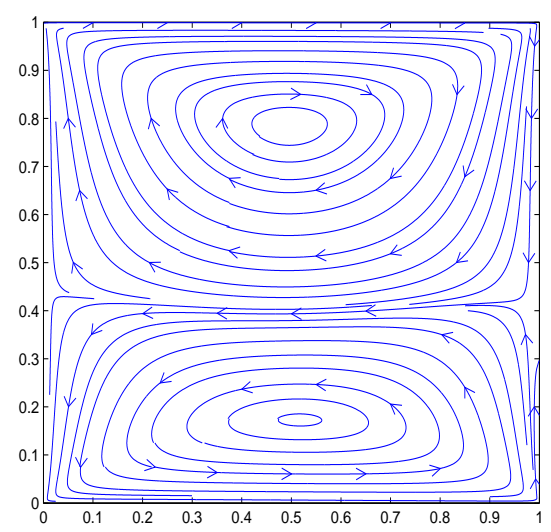

$(c)$

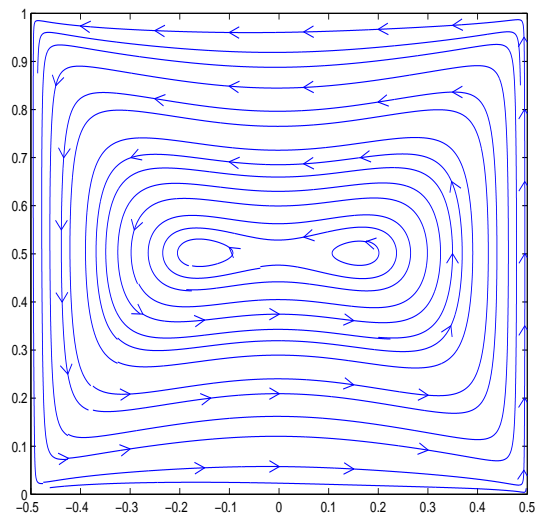

(b)

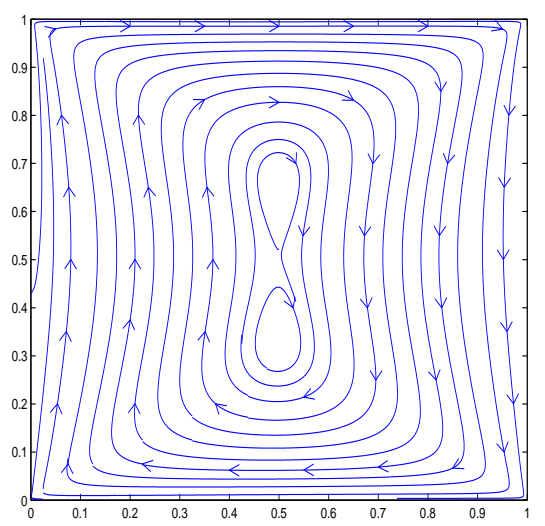

$(d)$

Figure 12: Validation of the DRBEM formulation 


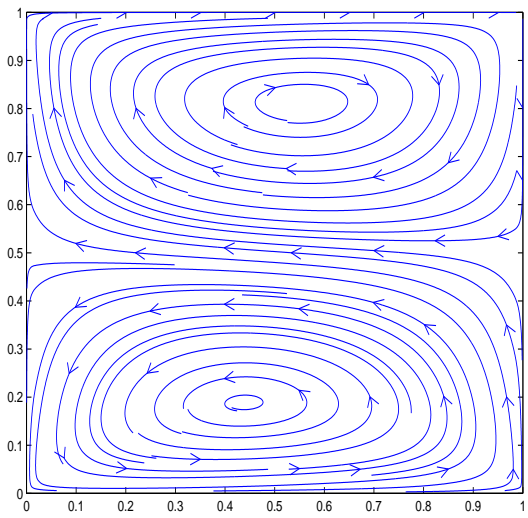

B. $E=120$

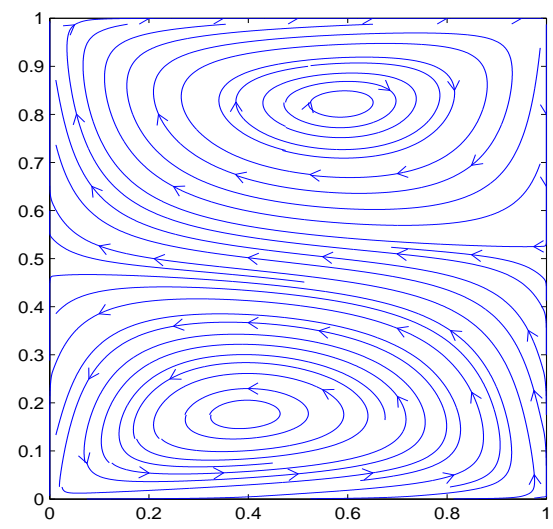

B. $E=320$

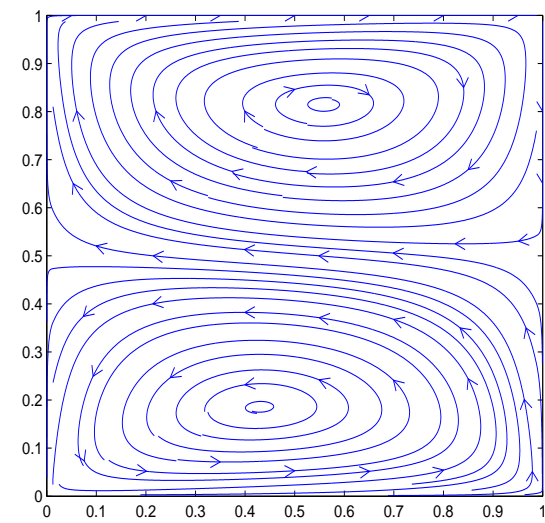

B. $E=160$

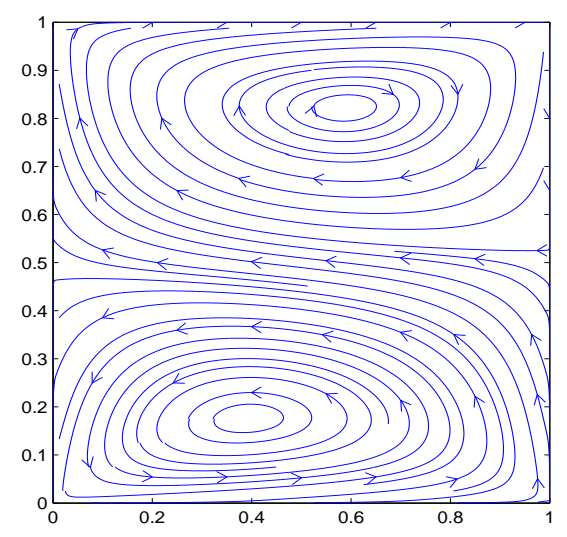

B. $E=360$

Figure 13: Effect of the number of boundary elements when $S=1$ and $L s=1$ 

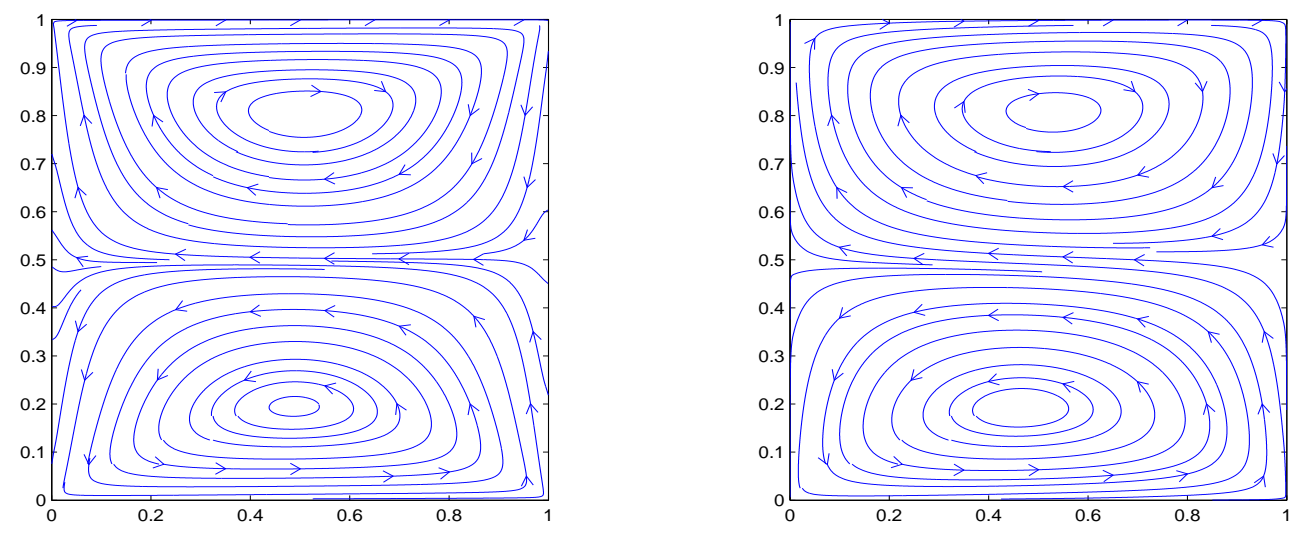

$$
L s=0
$$

$$
L s=0.25
$$
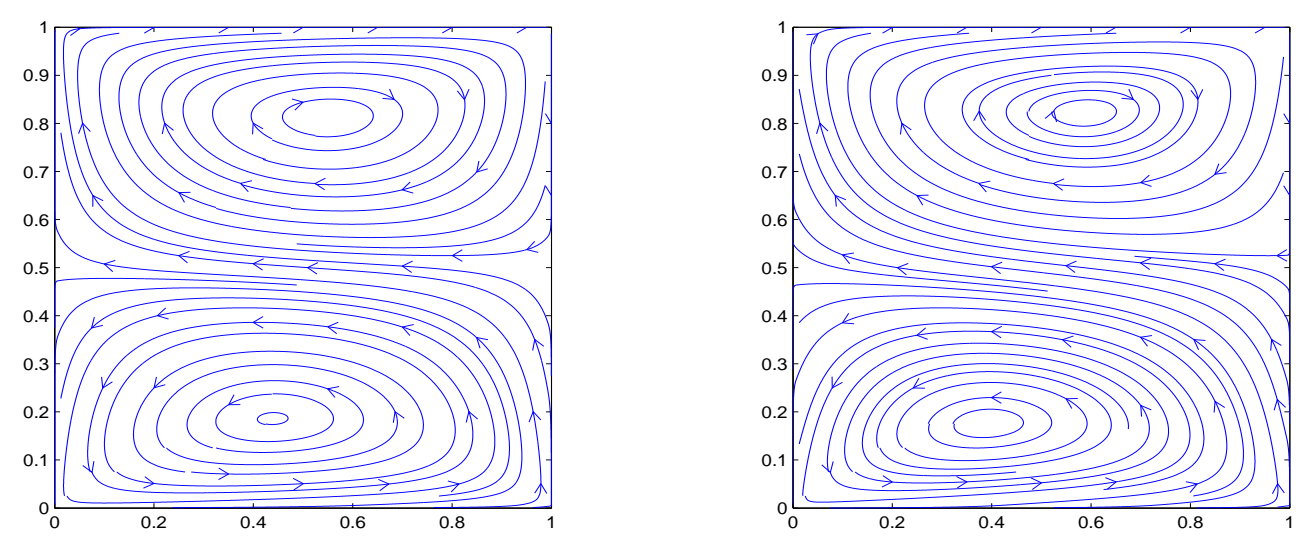

$$
L s=0.5
$$

$$
L s=1
$$

Figure 14: Upper and lower walls moving in the same direction, $S=1$ 


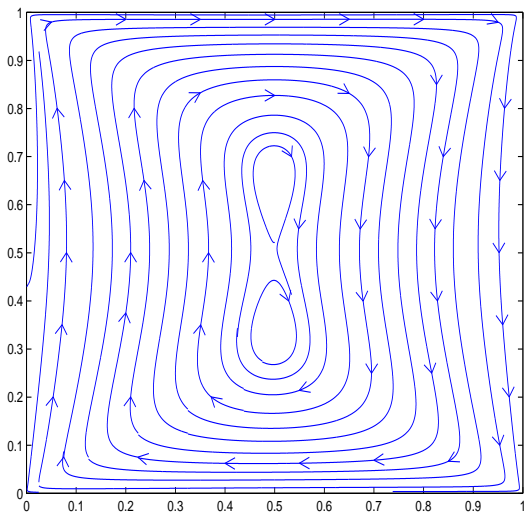

$$
L s=0
$$

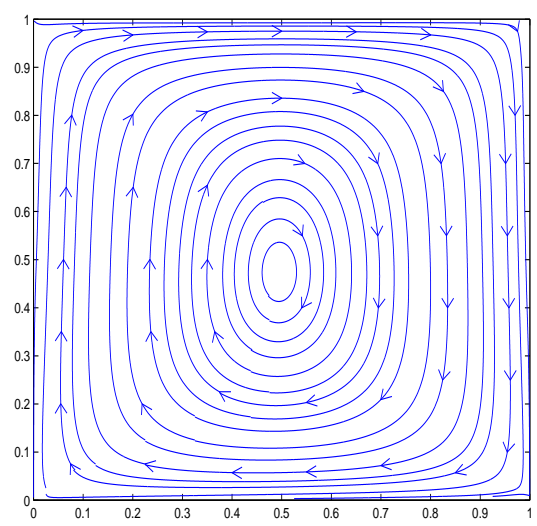

$$
L s=0.5
$$

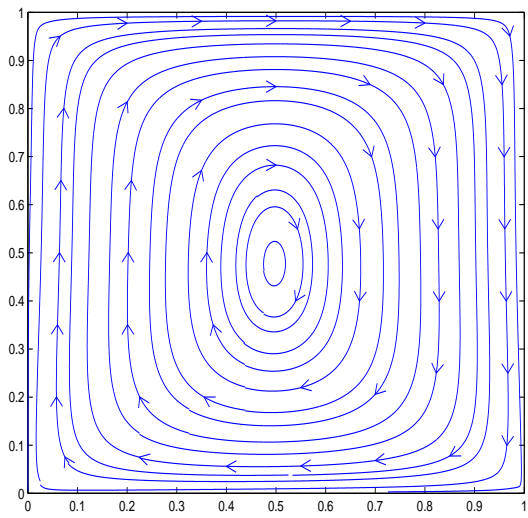

$L s=0.25$

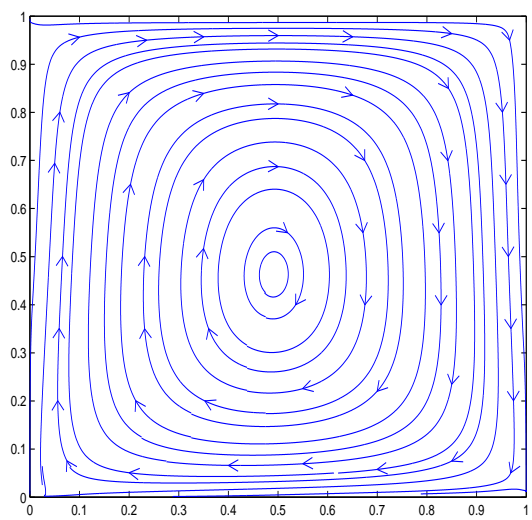

$$
L s=1
$$

Figure 15: Upper and lower walls moving in opposite directions, $S=-1$ 

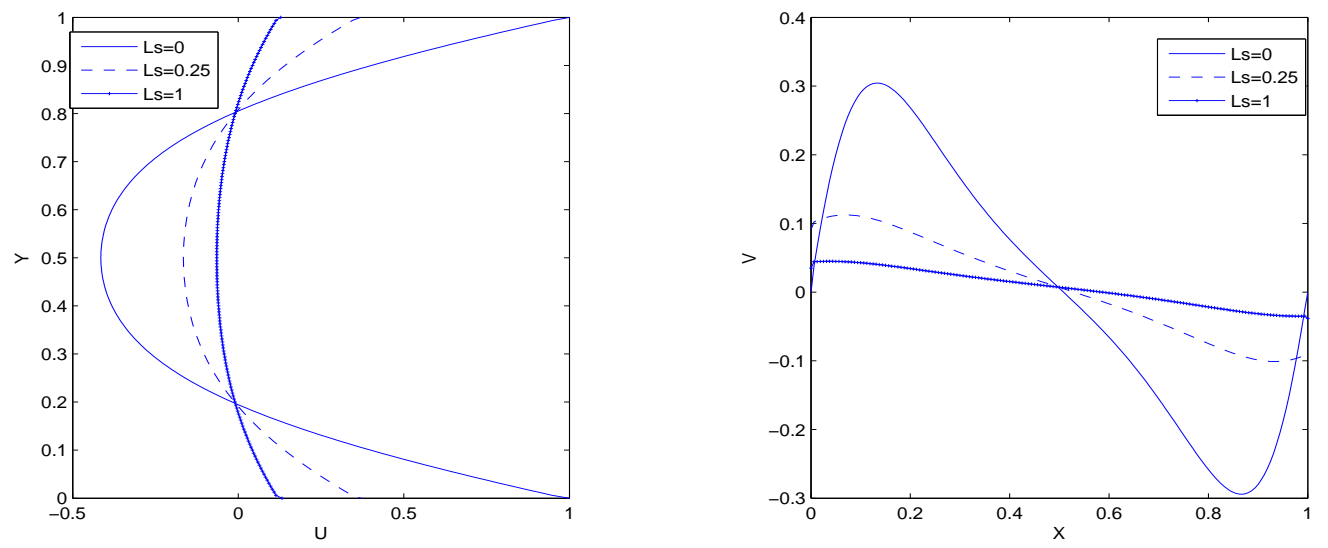

$$
S=1
$$
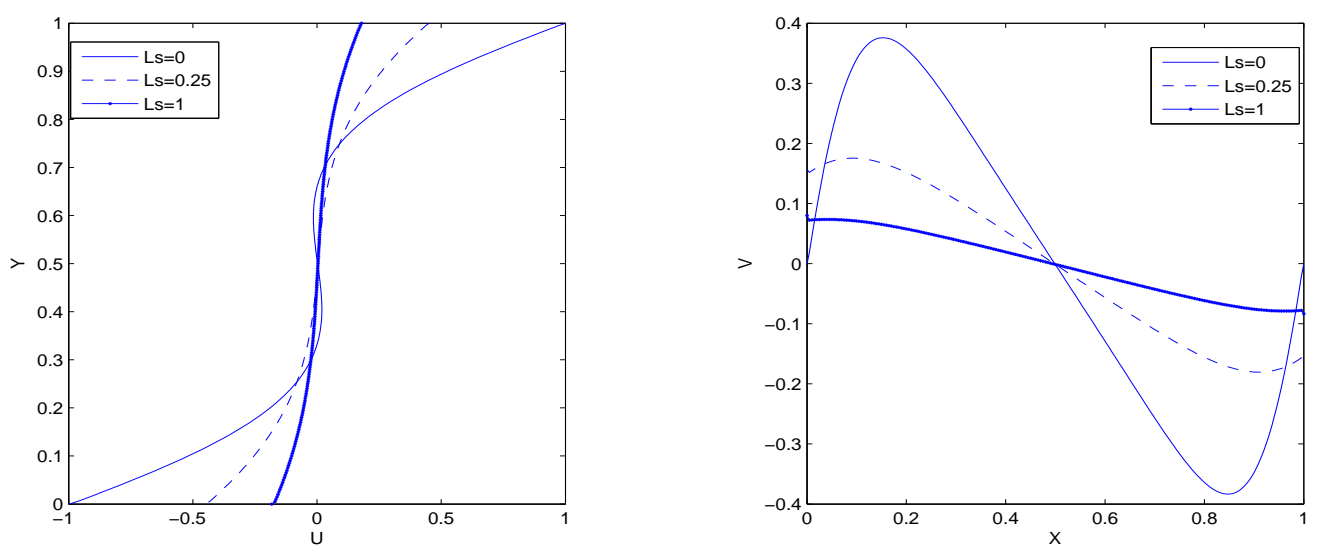

$$
S=-1
$$

Figure 16: Velocity profiles along the centerline for slip length values $0 \leq L s \leq 1$ 